\title{
Variant surface antigens of malaria parasites: functional and evolutionary insights from comparative gene family classification and analysis
}

\author{
Christian Frech and Nansheng Chen
}

\begin{abstract}
Background: Plasmodium parasites, the causative agents of malaria, express many variant antigens on cell surfaces. Variant surface antigens (VSAs) are typically organized into large subtelomeric gene families that play critical roles in virulence and immune evasion. Many important aspects of VSA function and evolution remain obscure, impeding our understanding of virulence mechanisms and vaccine development. To gain further insights into VSA function and evolution, we comparatively classified and examined known VSA gene families across seven Plasmodium species.
\end{abstract}

Results: We identified a set of ultra-conserved orthologs within the largest Plasmodium gene family pir, which should be considered as high-priority targets for experimental functional characterization and vaccine development. Furthermore, we predict a lipid-binding domain in erythrocyte surface-expressed PYST-A proteins, suggesting a role of this second largest rodent parasite gene family in host cholesterol salvage. Additionally, it was found that PFMC-2TM proteins carry a novel and putative functional domain named MC-TYR, which is conserved in other $P$. falciparum gene families and rodent parasites. Finally, we present new conclusive evidence that the major Plasmodium VSAs PfEMP1, SICAvar, and SURFIN are evolutionarily linked through a modular and structurally conserved intracellular domain.

Conclusion: Our comparative analysis of Plasmodium VSA gene families revealed important functional and evolutionary insights, which can now serve as starting points for further experimental studies.

Keywords: Malaria parasites, Plasmodium, Comparative genomics, Variant surface antigens, Gene family classification, Vaccine target

\section{Background}

Malaria is a major health problem in the world. Although global disease incidence is currently on decline, malaria remains responsible for at least 200 million infections and half a million deaths every year, in particular among immune-naïve African children under the age of five [1]. Human malaria is an infectious disease caused by five different species of eukaryotic parasites of the genus Plasmodium and is transmitted by Anopheles mosquitoes. Plasmodium falciparum is the most prevalent and virulent human malaria parasite accounting for almost all malarial deaths worldwide. Plasmodium vivax is the most prevalent

\footnotetext{
* Correspondence: chenn@sfu.ca

Department of Molecular Biology and Biochemistry, Simon Fraser University, 8888 University Drive, Burnaby, B.C V5A 156, Canada

thimon Fraser Uni
}

malaria parasite outside Africa where despite much lower mortality rates it represents a huge socioeconomic burden in many countries [2]. Plasmodium ovale and Plasmodium malariae cause a more benign form of human malaria and are responsible for only a small percentage of global infections. Plasmodium knowlesi, although traditionally considered a non-human parasite, is responsible for a potentially life-threatening zoonotic form of human malaria acquired from Southeast Asian macaque monkeys [3]. Other Plasmodium parasites are important model organisms in malaria research, including Plasmodium yoelii, Plasmodium chabaudi, and Plasmodium berghei (rodent parasites) as well as Plasmodium gallinaceum (bird parasite). A tree detailing the phylogenetic relationship of these and other Plasmodium species is provided in Additional file 1.

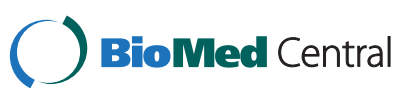

(c) 2013 Frech and Chen; licensee BioMed Central Ltd. This is an Open Access article distributed under the terms of the Creative Commons Attribution License (http://creativecommons.org/licenses/by/2.0), which permits unrestricted use, distribution, and reproduction in any medium, provided the original work is properly cited. 
One of the most pressing issues in malaria research is the development of an effective antimalarial vaccine. Unfortunately, despite many decades of research, this goal has yet to be achieved. RTS,S is currently the most promising $P$. falciparum vaccine candidate, but latest results from clinical trials showed that RTS,S provides only modest protection against both clinical and severe malaria in young infants [4,5]. For $P$. vivax the situation looks even grimmer with currently no vaccine candidate in advanced clinical trials [6]. In 2002, publication of the first two Plasmodium genomes, $P$. falciparum and $P$. yoelii, promised to revolutionize vaccine development by laying out the complete map of $P$. falciparum genes, including a comprehensive inventory of putative antigens that could serve as vaccine targets $[7,8]$. This monumental achievement was followed by the publication of the genomes of four additional Plasmodium species, including P. chabaudi and P. berghei in 2005 [9] as well as P. vivax and P. knowlesi in 2008 [10,11]. The P. gallinaceum genome was sequenced to three-fold coverage in 2007 and is currently unpublished. Although the availability of so many Plasmodium genome sequences provides now a rich resource for (comparative) genomics studies to learn more about parasite biology and immune evasion strategies $[12,13]$, the promise of an effective antimalarial vaccine has yet to be fulfilled.

One major obstacle in malaria vaccine development is the notorious variability of parasite antigens. These antigens are expressed at the surface of the parasite or of the infected erythrocyte and are typically encoded by large gene families located at subtelomeric regions of chromosomes. We now know that each of the sequenced Plasmodium genomes possesses an extensive and often species-specific array of variant surface antigens (VSAs) [14]. The clinically most important and best studied Plasmodium VSA gene family is var, which encodes for about 60 erythrocyte surface-expressed proteins known as $P$. falciparum erythrocyte membrane protein 1 (PfEMP1) [8]. In a process called antigenic variation, var gene expression switches from one family member to another over the course of an infection, allowing the parasite to evade the host immune system and to establish a chronic infection [15]. In addition, PfEMP1 mediates adherence of infected erythrocytes to both uninfected erythrocytes and endothelial cells, which is responsible for the most severe clinical complications of $P$. falciparum malaria and makes PfEMP1 the prime virulence factor in this species.

Besides PfEMP1, Plasmodium parasites express many additional VSAs throughout their complex life cycle. In $P$. falciparum, this includes the largest $P$. falciparum gene family rif/stevor ( 190 genes) [8,16], the surfin gene family (10 genes) [17], and Pfmc-2TM (12 genes) [18]. In $P$. vivax, the largest gene family by far is vir ( 300 genes) $[10,19]$, which is related to homologous VSA gene families named kir (65 genes) in P. knowlesi $[11,20]$ and yir ( 800 genes), bir ( 100 genes), and $\operatorname{cir}(\sim 200$ genes) in P. yoelii, $P$. berghei and P. chabaudi, respectively $[7,9,21]$. Together, these five gene families form the large pir superfamily, the largest known gene family in Plasmodium parasites [20]. To date no pir genes have been identified in P. falciparum. P. knowlesi possesses an additional large VSA gene family named SICAvar (28 genes), the first Plasmodium gene family demonstrated to undergo antigenic variation [11,22]. In rodent malaria parasites the second largest VSA gene family after pir is pyst- $a$, which in primate parasites consists of only a single member suggesting extensive expansion of this family in the rodent malaria species [7]. Besides VSAs, Plasmodium genomes encode another large repertoire of proteins termed the 'exportome' that is of potential interest for vaccine development [23]. Proteins in this set carry an N-terminal sequence motif termed Plasmodium export element (PEXEL) or vacuolar transport signal (VTS) that targets these proteins beyond the parasitophorous vacuole to the cytosol of the infected erythrocyte [24,25]. Exported proteins are then trafficked further to the erythrocyte surface or remain in the cytosol to help remodeling the infected host cell. Like VSAs, exported proteins are typically organized into subtelomeric variant gene families, most of which are species subset-specific [23]. Probably the most prominent exported gene family is the large and highly divergent gene family phist, which has 40-100 known members in each of the three primate parasites but only a single known member in rodent parasites [23].

Although their large numbers suggest that VSAs and exported proteins are of major importance for the parasite, we currently know surprisingly little about their biological functions. For example, proposed roles for PIR proteins include antigenic variation, immune evasion, signaling, trafficking, protein folding, and adhesion, but direct evidence for any PIR function is still lacking [14]. Similarly, apart from expression and localization information, the exact functions of PfMC-2TM, SURFIN, PYST-A, and PHIST proteins are currently unknown. Furthermore, the $P$. falciparum genome contains over a dozen exported gene families named hyp 1 to hyp 17 whose functions have yet to be determined. The difficulty in elucidating the function of these gene families is due in part to the presence of many functionally redundant paralogs, which makes gene knockout studies challenging. In such cases it would help if one can identify and work with low copy number orthologous gene families in more accessible model parasites. Besides unknown functions, the evolutionary history of many Plasmodium variant gene families is also poorly understood. For example, standard sequence similarity searches reveal no obvious homologs for the major surface antigens of $P$. falciparum (PfEMP1) and P. knowlesi (SICAvar) outside their respective species, raising the question about their evolutionary origin. Similarly, there 
are currently no known pir homologs in P. falciparum, although rif/stevor has been suggested as related gene family based on shared sequence motifs and secondary structural features [20]. The identification of functional homologs of VSAs across Plasmodium species is important because it aids comparative immunological studies, gives new insights into the evolutionary adaptation of malaria parasites to their respective hosts, and provides a means to transfer functional annotations from model to human parasites and vice versa.

In this study, we use a recently developed comparative gene family classification strategy [26] to classify VSAs and exported proteins across seven Plasmodium genomes, including P. falciparum, P. vivax, P. knowlesi, $P$. yoelii, P. chabaudi, P. berghei, and P. gallinaceum. We hypothesized that the sensitive sequence-based clustering of the entire body of currently available Plasmodium proteins will yield new insights into VSA function and evolution, which in turn could open up new avenues for vaccine development. In this strategy, protein sequences from Plasmodium genomes are first clustered into a hierarchical tree using average-linkage clustering and the resulting tree is then searched for clusters corresponding to known VSA gene families. Finally, identified clusters are closely analyzed for gene content and inter-cluster relationships. This analysis resulted in several noteworthy findings, including the identification of unusually well conserved PIR orthologs that are of potential interest for vaccine development, prediction of the likely function of PYST-A proteins, discovery of a novel and putatively functional PfMC-2TM domain named MC-TYR, new conclusive evidence supporting the common evolutionary origin of PfEMP1, SICAvar, and SURFIN proteins, and the identification of many new VSA gene family members, including new phist gene family members in rodent parasites. Collectively, these findings provide vital starting points for future experimental studies.

\section{Results}

\section{Curation and comparative classification of variant gene} families in Plasmodium genomes

We curated VSA and exported gene families by reviewing the literature as well as gene annotations in PlasmoDB 7.0 [27]. We were interested in all Plasmodium gene families that met one of the following criteria: (a) expressed at the parasite surface or the surface of infected erythrocytes; (b) predicted host cell localization by virtue of the presence of a PEXEL/VTS export motif; any other gene family that is (c) species (subset)-specific or (d) located at subtelomeric regions of chromosomes. Using these criteria, we compiled a list of 59 gene families (Table 1 and Additional file 2), which from now on we refer to as Plasmodium variant gene families.
After curation, we classified Plasmodium variant gene families using a comparative gene family classification strategy described previously [26]. Briefly, we hierarchically clustered the combined set of all protein sequences with MC-UPGMA [28] and extracted those sub-trees that maximally overlapped with the curated gene families. Most of the 59 curated gene families were resolved with both high sensitivity (Sn) and specificity (Sp) (Table 1). Average and median Jaccard indices (J) of extracted clusters were 0.87 and 0.98 , respectively. Almost half of gene families (28 gene families, 47\%) were clustered perfectly with a Jaccard index of 1.0. An additional 20 gene families (34\%) clustered with a good Jaccard index $\geq 0.75$. Only 11 gene families clustered with lower quality $(J<0.75)$ due to low sensitivity (PcEMA1, emp3, hyp 1, pk-fam-d), low specificity (TSP_1, hyp17, pv-fam-d, hyp6) or both (hyp15, TRAP, hyp2). Overall, we conclude that our clustering strategy works well on Plasmodium variant gene families and produces high-quality protein sequence clusters.

\section{PIR contains single ultra-conserved ortholog in each Plasmodium species}

The large pir gene family separates nicely into two large non-overlapping clusters of high sensitivity and specificity (Table 1). One cluster represents the vir and kir subfamilies of $P$. vivax and $P$. knowlesi, respectively (384 genes; $\mathrm{Sn}=94 \% ; \mathrm{Sp}=82 \% ; \mathrm{J}=78 \%$ ), and the other cluster represents the yir, bir, and cir subfamilies from P. yoelii, $P$. berghei, and $P$. chabaudi, respectively (1,192 genes; $\mathrm{Sn}=$ 99\%; Sp = 98\%; J = 97\%). The somewhat reduced specificity of the vir/kir gene cluster is due to inclusion of the $p v$ fam-c gene family (7 genes) that likely represents a bona fide vir subfamily (see below). In addition, the vir/kir cluster contains 59 P. vivax hypothetical proteins, many of which carry a predicted vir domain and are thus likely true but currently unannotated members of the vir gene family (see annotated gene models of the complete vir gene cluster at http://genome.sfu.ca/projects/gfc-plasmodium/).

To our surprise we found three rodent PIR proteins nested deep within the vir/kir cluster instead of clustering with their respective paralogs (Additional file 3). This unexpected result suggested that some of the otherwise highly divergent PIR proteins are well conserved across species. Subsequent comprehensive analysis of PIR sequence conservation revealed that each of the five Plasmodium species contains a single and likely orthologous PIR protein that is clearly better conserved between species than any other member of the PIR family (Figure 1A). The five genes are: P. vivax Vir14-related protein PVX_113230; P. knowlesi KIR protein PKH_114850; $P$. yoelii hypothetical protein PY06119; P. berghei BIR protein PBANKA_010050; and P. chabaudi CIR protein PCHAS_010120. Orthology of these five PIR proteins is supported by OrthoMCL DB (version 5.0, orthologous group OG5_173782) [29] and by 
Table 1 Plasmodium variant gene families and classification performance

\begin{tabular}{|c|c|c|c|c|c|c|c|c|c|c|}
\hline Gene family & Sn & $\mathrm{Sp}$ & Jacc & pfal & pviv & pkno & pyoe & pber & pcha & pgal \\
\hline \multicolumn{11}{|l|}{ VSA } \\
\hline var & 1.00 & 0.99 & 0.99 & $67(66)$ & 0 & 0 & 0 & 0 & 0 & 0 \\
\hline vir-kir & 0.94 & 0.82 & 0.78 & 1 & $310(262)$ & $66(65)$ & 3 & 2 & 2 & 1 \\
\hline yir-bir-cir & 0.99 & 0.98 & 0.97 & 0 & 0 & 0 & 897 & $100(99)$ & 195 (191) & 0 \\
\hline rif/stevor & 0.99 & 0.98 & 0.98 & $192(190)$ & 0 & 0 & 0 & 0 & 0 & 0 \\
\hline SICAvar & 1.00 & 0.90 & 0.90 & 0 & 0 & $31(28)$ & 0 & 0 & 0 & 0 \\
\hline Pfmc-2TM & 1.00 & 1.00 & 1.00 & $12(12)$ & 0 & 0 & 0 & 0 & 0 & 0 \\
\hline TryThrA-PVTRAG & 1.00 & 1.00 & 1.00 & $4(4)$ & $36(36)$ & 26 & 5 & 7 & 6 & 1 \\
\hline surfin/Pvstp1 & 1.00 & 1.00 & 1.00 & $9(9)$ & $2(2)$ & 0 & 0 & 0 & 0 & 2 \\
\hline cys6 & 1.00 & 1.00 & 1.00 & $10(10)$ & 11 & 12 & 14 & 10 & 10 & 6 \\
\hline P25_28 & 1.00 & 1.00 & 1.00 & $2(2)$ & $2(2)$ & $2(2)$ & $2(2)$ & $2(2)$ & $2(2)$ & 1 \\
\hline PCEMA1 & 0.71 & 0.92 & 0.67 & 0 & 0 & 0 & 2 & 1 & $13(17)$ & 0 \\
\hline pyst-a & 1.00 & 0.90 & 0.90 & 1 & 1 & 1 & 169 (138) & $23(22)$ & $132(131)$ & 1 \\
\hline \multicolumn{11}{|c|}{ VSA (invasion-linked) } \\
\hline$d b l$ & 1.00 & 1.00 & 1.00 & $5(5)$ & $2(2)$ & 4 & 2 & 2 & 2 & 1 \\
\hline$r b p / 235 \mathrm{kDa}$ & 1.00 & 1.00 & 1.00 & $5(5)$ & $8(8)$ & 1 & 19 & 6 & 8 & 0 \\
\hline$m s p-3$ & 0.89 & 0.89 & 0.80 & $4(6)$ & $14(12)$ & 4 & 2 & 2 & 2 & 0 \\
\hline$m s p-7$ & 1.00 & 1.00 & 1.00 & $6(6)$ & $11(11)$ & 4 & 3 & 3 & 3 & 1 \\
\hline rhoph1/clag & 1.00 & 1.00 & 1.00 & $5(5)$ & $3(3)$ & $1(1)$ & 2 & 2 & 3 & 1 \\
\hline TRAP & 0.60 & 0.75 & 0.50 & $4(5)$ & 4 & 3 & 4 & 4 & 4 & 3 \\
\hline$T S P_{-} 1$ & 1.00 & 0.73 & 0.73 & $8(6)$ & $8(7)$ & $7(5)$ & $9(5)$ & $8(5)$ & $8(7)$ & 5 \\
\hline PPLP & 1.00 & 1.00 & 1.00 & $5(5)$ & 5 & 5 & 5 & 5 & 5 & 3 \\
\hline
\end{tabular}

\section{Exported}

\begin{tabular}{|c|c|c|c|c|c|c|c|c|c|c|}
\hline phist/rad & 0.97 & 0.89 & 0.86 & $72(66)$ & 74 & 43 & 2 & 2 & 2 & 4 \\
\hline gbp130 & 1.00 & 1.00 & 1.00 & $3(3)$ & 0 & 0 & 0 & 0 & 0 & 0 \\
\hline pst-a & 0.97 & 1.00 & 0.97 & $10(10)$ & $9(10)$ & 6 & $12(12)$ & 4 & 28 & 1 \\
\hline emp3 & 0.50 & 1.00 & 0.50 & $1(2)$ & 1 & 1 & 0 & 0 & 0 & 0 \\
\hline ab_hyda & 1.00 & 1.00 & 1.00 & $4(4)$ & 2 & 2 & 2 & 2 & 2 & 1 \\
\hline$a b \_h y d b$ & 1.00 & 1.00 & 1.00 & $4(4)$ & 1 & 1 & 0 & 0 & 0 & 1 \\
\hline HRP & 1.00 & 1.00 & 1.00 & $2(2)$ & 0 & 0 & 0 & 0 & 0 & 0 \\
\hline hyp 1 & 0.50 & 1.00 & 0.50 & $1(2)$ & 0 & 0 & 0 & 0 & 0 & 0 \\
\hline hyp2 & 0.50 & 0.50 & 0.33 & $2(2)$ & 0 & 0 & 0 & 0 & 0 & 0 \\
\hline hyp4 & 1.00 & 1.00 & 1.00 & $9(9)$ & 0 & 0 & 0 & 0 & 0 & 0 \\
\hline hyp5 & 1.00 & 0.89 & 0.89 & $9(8)$ & 0 & 0 & 0 & 0 & 0 & 0 \\
\hline hyp6 & 1.00 & 0.40 & 0.40 & $5(2)$ & 0 & 0 & 0 & 0 & 0 & 0 \\
\hline hyp7 & 1.00 & 1.00 & 1.00 & $3(3)$ & 0 & 0 & 0 & 0 & 0 & 0 \\
\hline hyp8 & 1.00 & 1.00 & 1.00 & $2(2)$ & 0 & 0 & 0 & 0 & 0 & 0 \\
\hline hyp9 & 1.00 & 1.00 & 1.00 & $5(5)$ & 0 & 0 & 0 & 0 & 0 & 0 \\
\hline hyp10 & 1.00 & 1.00 & 1.00 & $2(2)$ & 0 & 0 & 0 & 0 & 0 & 0 \\
\hline hyp 11 & 1.00 & 1.00 & 1.00 & $5(5)$ & 6 & 5 & 1 & 1 & 1 & 0 \\
\hline hyp 12 & 1.00 & 0.75 & 0.75 & $4(3)$ & 0 & 0 & 0 & 0 & 0 & 0 \\
\hline hyp 13 & 1.00 & 1.00 & 1.00 & $2(2)$ & 0 & 0 & 0 & 0 & 0 & 0 \\
\hline hyp 15 & 0.75 & 0.75 & 0.60 & $4(4)$ & 0 & 0 & 0 & 0 & 0 & 0 \\
\hline
\end{tabular}


Table 1 Plasmodium variant gene families and classification performance (Continued)

\begin{tabular}{|c|c|c|c|c|c|c|c|c|c|c|}
\hline Gene family & Sn & $\mathrm{Sp}$ & Jacc & pfal & pviv & pkno & pyoe & pber & pcha & pgal \\
\hline hyp16 & 1.00 & 1.00 & 1.00 & $2(2)$ & 0 & 0 & 0 & 0 & 0 & 0 \\
\hline hyp 17 & 1.00 & 0.67 & 0.67 & $3(2)$ & 0 & 0 & 0 & 0 & 0 & 0 \\
\hline$p k$-fam-b & 1.00 & 0.83 & 0.83 & 0 & 1 & $12(10)$ & 0 & 0 & 0 & 0 \\
\hline$p k$-fam-c & 1.00 & 1.00 & 1.00 & 0 & 0 & $5(5)$ & 0 & 0 & 0 & 0 \\
\hline pk-fam-e & 1.00 & 1.00 & 1.00 & 0 & 0 & $3(3)$ & 0 & 0 & 0 & 0 \\
\hline \multicolumn{11}{|c|}{ Other (species-specific or sub-telomeric) } \\
\hline etramp & 0.97 & 0.94 & 0.91 & $15(14)$ & $10(9)$ & 9 & 15 & $6(7)$ & 12 & 4 \\
\hline acs & 1.00 & 0.93 & 0.93 & $14(13)$ & 5 & 5 & 5 & 5 & 7 & 5 \\
\hline$A C B P$ & 1.00 & 1.00 & 1.00 & $4(4)$ & 0 & 0 & 0 & 0 & 0 & 0 \\
\hline$p v-f a m-g$ & 1.00 & 1.00 & 1.00 & 3 & $3(3)$ & 3 & 3 & 3 & 3 & 3 \\
\hline$p k$-fam-a & 1.00 & 0.89 & 0.89 & 0 & 0 & $9(8)$ & 0 & 0 & 0 & 0 \\
\hline$p c$-fam & 0.85 & 1.00 & 0.85 & 0 & 0 & 0 & 5 & 1 & $17(20)$ & 0 \\
\hline pk-fam-d & 0.50 & 1.00 & 0.50 & 0 & 0 & $1(2)$ & 0 & 0 & 0 & 0 \\
\hline$p v-f a m-d$ & 1.00 & 0.57 & 0.57 & 1 & $28(16)$ & 9 & 0 & 0 & 0 & 0 \\
\hline pyst-c & 1.00 & 0.82 & 0.82 & 0 & 0 & 0 & $22(18)$ & 3 & 11 & 0 \\
\hline$p v-f a m-b$ & 1.00 & 1.00 & 1.00 & 0 & $6(6)$ & 1 & 0 & 0 & 0 & 0 \\
\hline$p v-f a m-c$ & 1.00 & 1.00 & 1.00 & 0 & 7 (7) & 0 & 0 & 0 & 0 & 0 \\
\hline pyst-d & 0.77 & 1.00 & 0.77 & 0 & 0 & 0 & $10(13)$ & 0 & 0 & 0 \\
\hline pyst-b & 1.00 & 0.96 & 0.96 & 0 & 0 & 0 & $56(54)$ & 28 & 21 & 0 \\
\hline$p v-f a m-h$ & 1.00 & 0.80 & 0.80 & 4 & $5(4)$ & 3 & 0 & 0 & 0 & 0 \\
\hline Average & 0.94 & 0.92 & 0.87 & & & & & & & \\
\hline
\end{tabular}

Fifty-nine Plasmodium variant gene families were curated from the literature and public databases and categorized as follows: (a) VSAs (11 families); (b) VSAs linked to erythrocyte invasion ( 8 families); (c) predicted to be exported to the host erythrocyte ( 25 families); (d) other subtelomeric or species-specific gene families (15 families). Better studied gene families are shown at the top of each category. Classification performance of each gene family is measured in terms of sensitivity (Sn), specificity (Sp), and Jaccard index (Jacc). The remaining columns show how many genes of each species have been classified to belong to this gene family (excluding annotated pseudogenes and gene fragments). Numbers in parentheses indicate how many genes of each species served as reference gene family members. A more comprehensive version of this table including numbers of identified genes in non-Plasmodium species is available in Additional file 2. Abbreviations: VSA... variant surface antigen; pfal... P. falciparum; pviv... P. vivax; pkno... P. knowlesi; pyoe... P. yoelii; pber... P. berghei; pcha... P. chabaudi; pgal... P. gallinaceum.

the fact that gene synteny is conserved among the five species (PlasmoDB 9.3). Multiple sequence alignment of the five conserved PIR orthologs reveals a 224 aa long, gapless block recognized as the Plasmodium_Vir domain (PF05795). The block spans 56\% of the average sequence length and has 107 columns (48\%) perfectly conserved (Figure 1B). This exceptional high degree of sequence conservation within an otherwise highly divergent gene family suggests that these genes are direct descendants of the founder member of the large pir gene family and that they serve an ancestral and probably special function in malaria parasites.

Identification of the likely prototypical members of the large pir gene family allowed us to re-examine whether there exists conserved PIR homologs outside $P$. vivax/P. knowlesi and rodent malaria parasites, in particular within the Laverania clade including P. falciparum. NCBI PSIBLAST search (http://blast.ncbi.nlm.nih.gov) seeded with the five conserved PIR orthologs did not reveal significant sequence similarity within $P$. falciparum or any other species not currently known to contain PIR proteins. Similarly, multiple rounds of Jackhmmer searches [30] seeded with the alignment from Figure 1 did not identify convincing homologs. Thus, despite extensive sequence conservation between the primate and rodent parasites, PIR is apparently not conserved in other Plasmodium or Apicomplexan species, suggesting that PIR surface antigens perform an important function unique to the $P$. vivax/P. knowlesi and rodent clade of malaria parasites.

\section{PYST-A proteins predicted to be involved in lipid binding and transfer}

Gene family expansion is an important genomic process by which parasites adapt to different lifestyles and host environments [31,32]. Our classification strategy readily identifies differentially expanded gene families in several Plasmodium species (Table 1), including rhoph1/clag, surfin and acs (expanded in P. falciparum), msp-3, msp7 , and pv-fam-d (expanded in P. vivax), TryThrA/ 


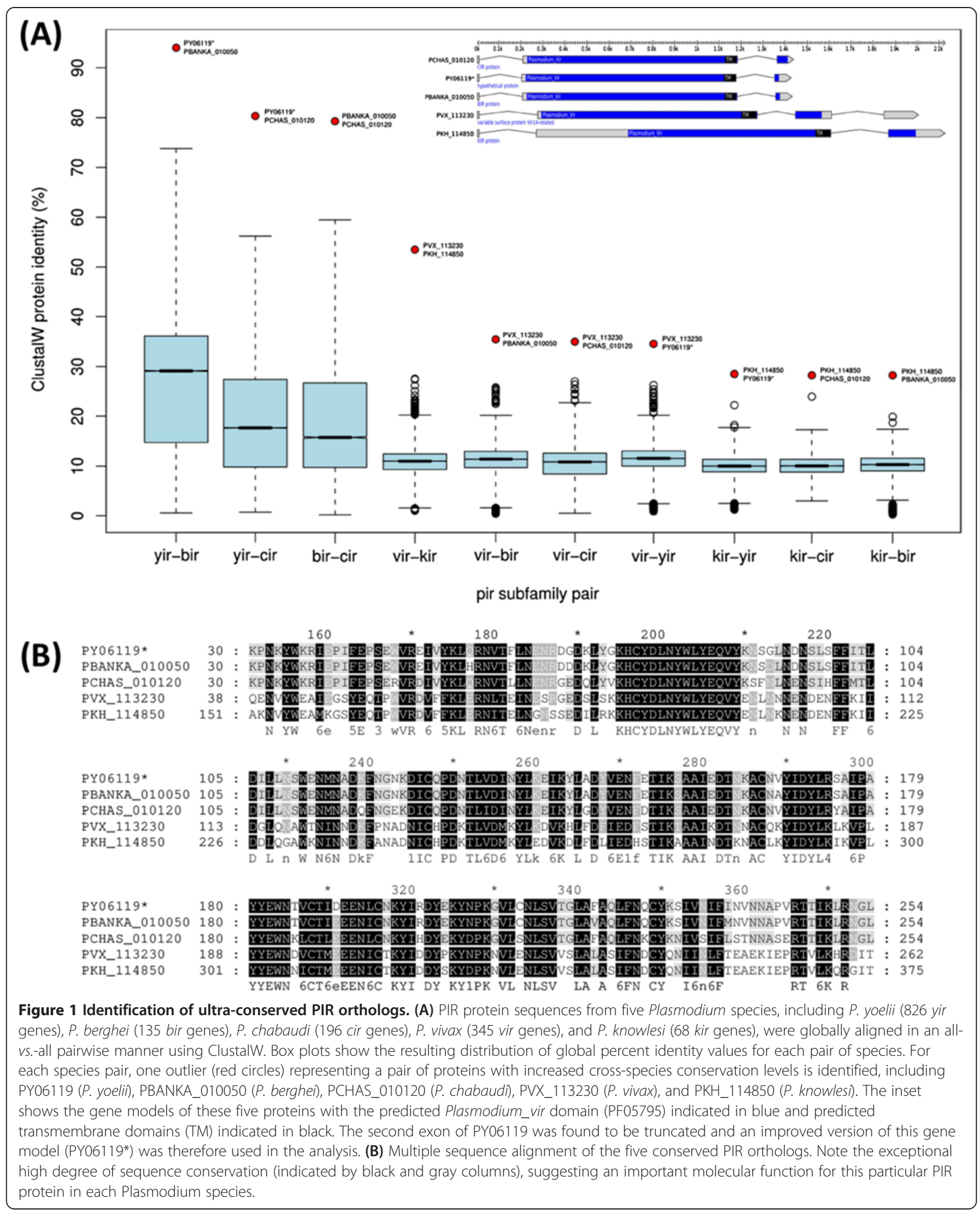


PVTRAG (expanded in P. vivax and P. knowlesi), phist and hyp11 (expanded in the three primate parasites), $r b p / 235 k D a$ and pyst-c (expanded in P. yoelii), and PcEMA1, pc-fam, and pst-a (expanded in P. chabaudi).

One of the most striking examples of a differentially expanded gene family in Plasmodium parasites is the P. yoelii gene family pyst-a (Table 1). Pyst- $a$ has been shown to contain only a single member in primate malaria parasites but over one hundred members in P. yoelii and P. chabaudi $[7,9]$. In our analysis, PYST-A proteins cluster almost perfectly $(\mathrm{Sn}=100 \%$; $\mathrm{Sp}=90 \%$; $=90 \%)$ within a larger cluster containing also PC-FAM-1 and PB-FAM-1 proteins, clearly showing that these three gene families are equivalent (orthologous) in the three rodent parasite species. Total gene numbers in the combined pyst-a/pc-fam-1/pb-fam-1 sequence cluster are 168, 132, 23 genes in $P$. yoelii, $P$. chabaudi, and P. berghei, respectively. As expected, we found only a single pyst- $a$ gene family member in primate malaria parasites (PF14_0604 in P. falciparum, PVX_117290 in P. vivax, and PKH_124210 in P. knowlesi). A single copy of pyst- $a$ was also detected in the bird parasite $P$. gallinaceum, supporting the idea of extensive proliferation of the pyst-a gene family in the rodent malaria parasite lineage [7].

Interestingly, while examining annotated pyst-a gene models (Figure 2A), we noticed that most pyst-a gene family members carry a predicted Bet v1-like superfamily domain [33] (http://supfam.org/SUPERFAMILY/cgibin/scop.cgi?ipid=55961; HMMER3 E-value $\leq 1 \mathrm{e}-10$ ), suggesting a role of PYST-A proteins in lipid transport. In search for further bioinformatics support for this possibility, we used I-TASSER to predict the tertiary and secondary structure of $P$. falciparum gene family member PF14_0604 (Figure 2B and Figure 2C). The predicted protein structure is very similar to several human proteins carrying the steroidogenic acute regulatory-related lipid transfer (START) domain, including PDB protein 1EM2 $(\mathrm{C}$-score $=-1.22 ;$ TM-score $=0.724 ; \mathrm{RMSD}=1.71)$. Taken together, our bioinformatics analysis suggests that the second largest rodent malaria parasite gene family pyst-a is involved in lipid binding and transport, probably playing a role in host cholesterol salvage (see Discussion).

\section{PfMC-2TM proteins are conserved in rodent parasites and related to HYP8 and HYP2 through the novel MC-TYR domain}

Hierarchical protein sequence clustering is a powerful approach to elucidate distant evolutionary relationships between gene families [34]. Table 2 summarizes known and novel evolutionary links between Plasmodium variant gene families as suggested by hierarchical clustering. Novel links of note include links between Pfmc-2TM and hyp 8 (BLAST match pair percentage $(\mathrm{MP})=100 \%$; average E-value of BLAST match pairs $(\mathrm{E})=1.6)$, Pfmc-
2TM/hyp8 and hyp2 (MP =76.7\%; $\mathrm{E}=5.6)$, hyp4 and hyp6 $(\mathrm{MP}=80 \%, \mathrm{E}=1.9)$, hyp5 and hyp15 $(\mathrm{MP}=100 \%$, $\mathrm{E}=0.082)$, hyp16, pv-fam- $h$, and pk-fam-c $(\mathrm{MP}=30.6 \%$, $\mathrm{E}=0.72)$, as well as $p k-f a m-a, p k-f a m-b$ and $p v-f a m-d$ ( $\mathrm{MP}=11.6 \%, \mathrm{E}=0.048)$. Thus, several Plasmodium variant gene families currently assumed to be species-specific have in fact putative members in other Plasmodium species, opening up new avenues for elucidating gene function. Of note, we found that $p v-f a m-c$ is entirely nested within the larger vir/kir cluster $(\mathrm{MP}=2.9 \%, \mathrm{E}=$ 1.3) and thus likely represents a novel vir subfamily that we named vir36. pyst-d (InterPro IPR006492), a family of $\sim 15$ short (median length 60 aa) P. yoelii proteins, was found to be completely nested within the large pir gene family. Inspection of pyst- $d$ and neighboring gene models revealed that this gene family is likely spurious and consists entirely of misannotated exons belonging to adjacent yir gene models. Other novel links in Table 2 are of lower connectivity and should be regarded as tentative.

Pfmc-2TM is an actively studied P. falciparum VSA gene family $[18,35,36]$, which motivated us to investigate its putative link with the two P. falciparum hypothetical gene families hyp 8 and hyp2 in detail. The 12 annotated PfMC-2TM proteins cluster first with two P. falciparum gametocyte-exported proteins of the hyp 8 gene family (MAL13P1.61/GEXP07 and PFA0670c/GEXP10) and then with a cluster of three $P$. falciparum exported proteins of unknown function, one of which is a member of the hyp2 gene family (PFB0926c). The second annotated hyp2 gene family member (PF10_0024) is not found in this cluster and instead groups with hyp16 and $p v$-fam- $h$ proteins, supporting the previously expressed idea that hyp 2 does not constitute a real gene family [23]. The combined Pfmc$2 T M /$ hyp $8 /$ hyp 2 cluster contains a total of 23 proteins and is shown in Figure 3A. Notably, this cluster also includes six rodent parasite genes of unknown function that share the typical two-exon gene structure with $P f m c-2 T M$ genes, including a signal peptide on the short first exon and a conserved PEXEL-like motif (RxLxQ) on the $5^{\prime}$ end of the larger second exon. Most importantly, Phobius [37] predicts two adjacent C-terminal transmembrane (TM) domains for four of the six rodent parasite proteins (posterior probability $>0.2$ ) as well as traces of a second TM domain in the remaining two (posterior probability $<0.2$ ). No TM domains are present in the three $P$. falciparum proteins clustering with (and including) HYP2 protein PFB0926c.

Further inspection of the Pfmc-2TM/hyp8/hyp2 gene cluster using multiple protein sequence alignments identified a hitherto unknown $\sim 70$ aa long conserved domain located between the PEXEL motif and the two TM domains (Figure 3B). The domain has a predicted threealpha helical structure and contains two almost invariant tyrosine residues $(\mathrm{Y})$ of potential functional importance. Only in three proteins, one of the two tyrosine residues 


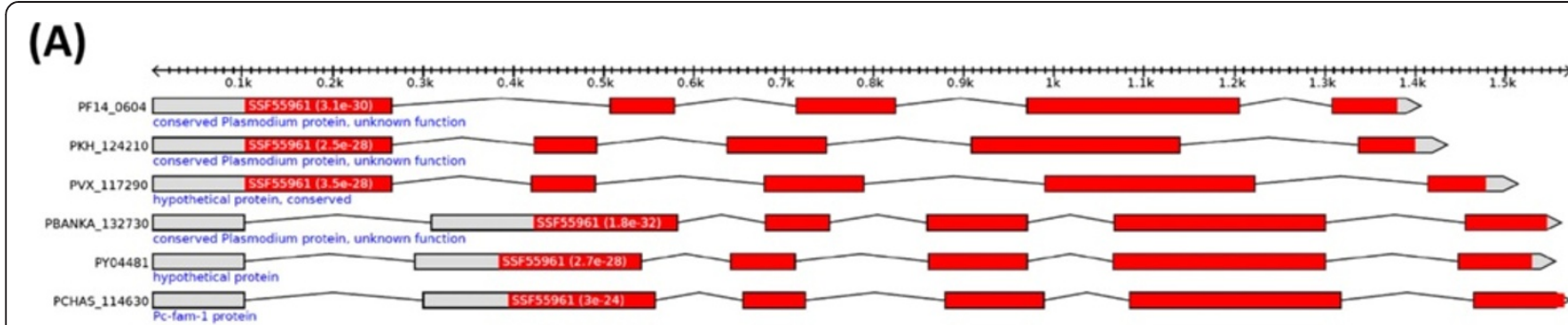

(B)

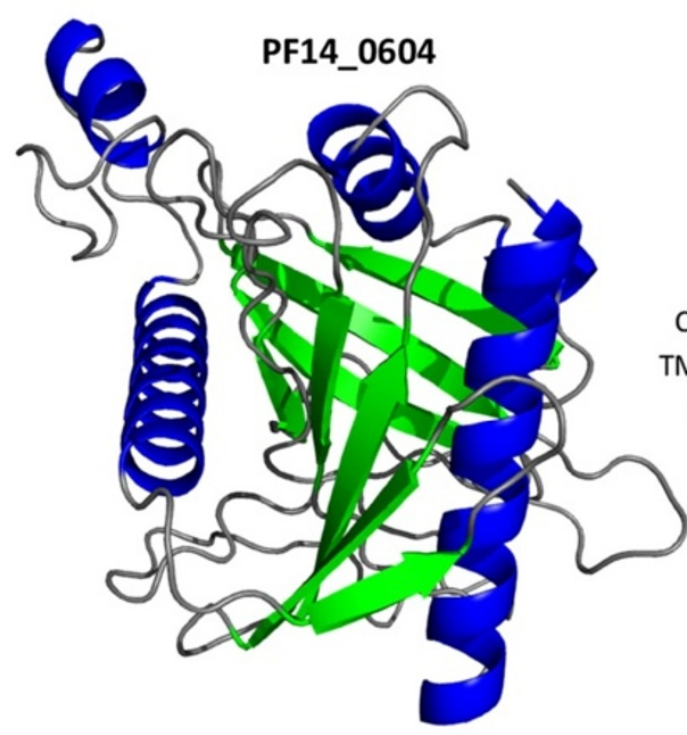

C-score $=-1.22$

TM-score $=0.724$

$\mathrm{RMSD}=1.71$

$\mathrm{PID}=12.5$

(C)

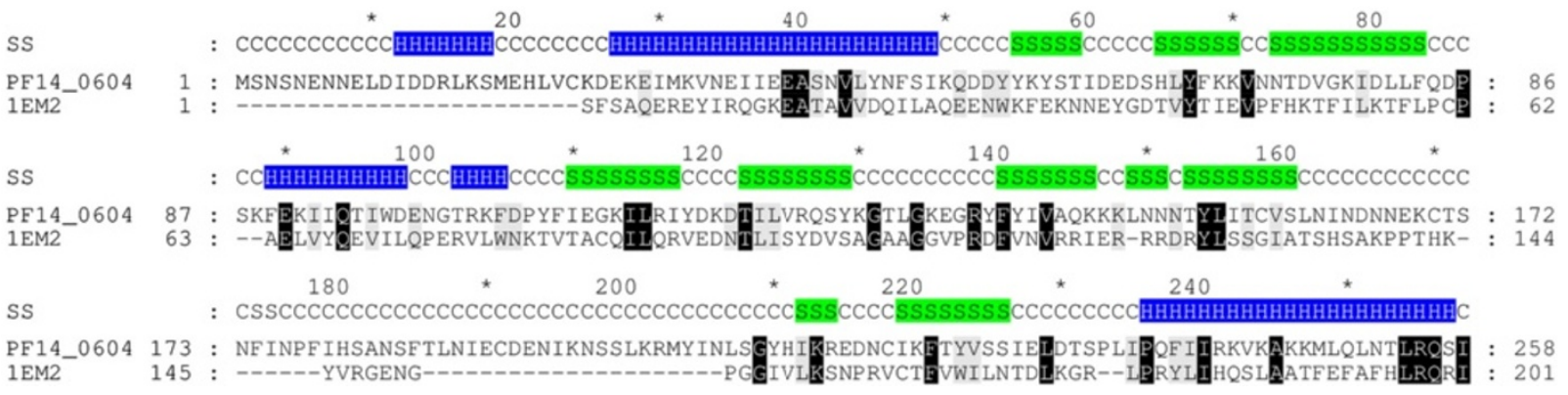

Figure 2 PYST-A proteins predicted to be involved in lipid binding and transfer. (A) Predicted Bet v1-like superfamily domain (SSF55961, in red) in selected pyst-a gene family members from six Plasmodium species. Hmmer3 E-values shown in parentheses. (B) I-TASSER predicted homology model (C-score $=-1.22)$ of the single $P$. falciparum pyst-a gene family member PF14_0604 (left) next to crystal structure of steroidogenic acute regulatory-related lipid transfer (START) domain-containing human protein MLN64 (PDB entry 1EM2; right). Predicted alpha helices and beta strands of PF14_0604 highlighted in blue and green, respectively. Note the overall high similarity between the two structures $(T M-s c o r e=0.724 ; \mathrm{RMSD}=1.71)(\mathbf{C})$ Protein sequence alignment obtained by threading PF14_0604 onto 1 EM2 using MUSTER. Percent sequence identity (PID) of structurally aligned residues is $12.5 \%$ over $78 \%$ of PF14_0604 sequence length. Secondary structure (SS) elements colored according to structural model in B. Identical and similar amino acids colored in black and gray, respectively. Sequence and structural similarity suggest a potential role of the pyst-a gene family in lipid binding and transfer.

is conservatively replaced with phenylalanine (F). Based on this observation, we name this novel domain the Maurer's clefts tyrosine (MC-TYR) domain.

To explore the possibility that MC-TYR is conserved beyond the seed cluster shown in Figure 3, we carried out sensitive profile-based searches using PSI-BLAST. Five rounds of NCBI PSI-BLAST initiated with any one of the
12 Pfmc-2TM MC-TYR protein sequences consistently identified three additional $P$. falciparum hits, albeit above PSI-BLAST's inclusion threshold $(\mathrm{E}>0.005)$. These three hits include one of three annotated HYP12 proteins (MAL7P1.6; $\mathrm{E}=0.2$ ) and two other exported proteins of unknown function (PF11_0511 and MAL8P1.206; E $\leq 2.7$ ). Like the three proteins of the hyp 2 cluster, these three 
Table 2 Established and novel evolutionary links between Plasmodium variant gene families predicted by hierarchical clustering

\begin{tabular}{|c|c|c|c|c|c|c|c|}
\hline Cluster A & Size $A$ & Cluster B & Size B & MP\# & MP\% & Avg. Evalue & Avg. PID(Stddev) \\
\hline \multicolumn{8}{|l|}{ Established links } \\
\hline rif & 161 & stevor & 31 & 281,244 & $74.8 \%$ & 0.016 & $26.4(3.1)$ \\
\hline vir/kir & 385 & yir/bir/cir & 1,192 & 2,087 & $0.4 \%$ & 9.7 & $28.2(5.2)$ \\
\hline surfin & 15 & Pvstp1 & 2 & 90 & $93.8 \%$ & $3.9 e-15$ & $38.3(6.6)$ \\
\hline var & 67 & $d b l$ & 18 & 707 & $42.8 \%$ & $1.7 e-10$ & $25.0(2.9)$ \\
\hline phist-c & 80 & $\mathrm{rad}$ & 58 & 718 & $15.5 \%$ & 0.38 & $23.9(3.7)$ \\
\hline phist-c/rad & 138 & phist-b & 21 & 241 & $8.3 \%$ & 2.4 & $25.2(4.2)$ \\
\hline phist-c/rad/phist-b & 168 & phist-a & 29 & 273 & $4.0 \%$ & 1.6 & $26.4(5.3)$ \\
\hline$a b \_h y d b$ & 10 & pst-a & 77 & 787 & $99.6 \%$ & $1.7 e-11$ & $33.1(6.2)$ \\
\hline$a b \_h y d b / p s t-a$ & 87 & ab_hyda & 17 & 231 & $10.4 \%$ & 3.8 & $28.4(5.5)$ \\
\hline surfin/Pvstp1 & 17 & SICAvar & 31 & 2,003 & $35.7 \%$ & 0.013 & $37.8(8.4)$ \\
\hline surfin/Pvstp1/SICAvar & 51 & var & 67 & 435 & $1.8 \%$ & 1.9 & $37.9(6.8)$ \\
\hline \multicolumn{8}{|l|}{ Novel links } \\
\hline surfin/Pvstp 1/SICAvar/var & 149 & pir & 1,814 & 772 & $0.1 \%$ & 14 & $30.5(6.5)$ \\
\hline Pfmc-2TM & 12 & hyp8 & 2 & 24 & $100.0 \%$ & 1.6 & $27.9(2.4)$ \\
\hline Pfmc-2TM/hyp8 & 14 & hyp2 & 2 & 23 & $76.7 \%$ & 5.6 & $32.6(2.8)$ \\
\hline hyp4 & 9 & hyp6 & 5 & 40 & $80.0 \%$ & 1.9 & $26.7(1.5)$ \\
\hline hyp15 & 4 & hyp5 & 9 & 44 & $100.0 \%$ & 0.082 & $31.0(5.5)$ \\
\hline pk-fam-a & 9 & $p k$-fam-b & 13 & 38 & $16.2 \%$ & $2.3 e-06$ & $55.7(18.4)$ \\
\hline$p k$-fam-a/pk-fam-b & 22 & $p v$-fam-d & 38 & 137 & $11.6 \%$ & 0.048 & $30.6(7.9)$ \\
\hline pyst-a/pc-fam-1/pb-fam-1 & 328 & PCEMA1 & 16 & 90 & $1.5 \%$ & 7.7e-32 & $50.6(8.9)$ \\
\hline$p v-f a m-h^{*}$ & 12 & hyp 16 & 2 & 16 & $80.0 \%$ & $7.8 \mathrm{e}-12$ & $33.7(6.8)$ \\
\hline hyp 16/pv-fam-h & 12 & $p k$-fam-c & 5 & 22 & $30.6 \%$ & 0.72 & $38.1(5.5)$ \\
\hline$T S P_{-} 1$ & 53 & $P 25 / 28$ & 13 & 29 & $1.1 \%$ & 18 & $32.0(11.2)$ \\
\hline hyp 11 & 19 & $\mathrm{rbp} / 235 \mathrm{kDa}$ & 47 & 13 & $1.1 \%$ & 31 & $30.4(6.0)$ \\
\hline phist-c/rad/phist-b* & 168 & pk-fam-e & 3 & 30 & $4.5 \%$ & $1.9 e-06$ & $67.5(14.3)$ \\
\hline vir/kir* & 385 & $p v-f a m-c$ & 7 & 94 & $2.9 \%$ & 1.3 & $27.0(5.2)$ \\
\hline pir* $^{*}$ & 1,814 & pyst-d & 10 & 179 & $0.9 \%$ & 0.11 & $64.2(14.6)$ \\
\hline
\end{tabular}

The hierarchical tree was searched for neighboring and parental clusters where both clusters (denoted A and B) map to variant gene families as identified in Table 1. Such cluster pairs are shown if (a) their BLAST match pair percentage (MP\%) is $\geq 1 \%$ or (b) they share at least 100 BLAST match pairs (MP\#). Avg. E-value and Avg. PID denote the average E-value and percent identity of all BLAST match pairs between the two clusters. Size $A$ and Size $B$ indicate numbers of genes in respective clusters, excluding annotated pseudogenes and gene fragments. An asterisk next to cluster $A$ indicates that it is a parental cluster of cluster $B$, i.e. cluster B is fully contained in cluster A. Our search predicts both previously established (top) and novel evolutionary links (bottom) between Plasmodium variant gene families.

genes share the typical two-exon gene structure with Pfmc-2TM genes except for the TM domains. This data suggests that MC-TYR is likely conserved in additional exported $P$. falciparum proteins and probably includes the $P$. falciparum hyp12 gene family. We should mention that neither protein sequence clustering nor PSI-BLAST searches identified a relationship of PfMC-2TM with HYP7, PYST-B or virD proteins, which has been proposed previously based on shared gene architectural features $[18,38]$.

Taken together, we provide first evidence that PfMC2TM proteins carry a novel and putative functional domain named MC-TYR. MC-TYR is conserved in other P. falciparum exported proteins and additional Plasmodium species, opening up new opportunities to experimentally characterize the function of this important VSA gene family.

PfEMP1, SICAvar, and SURFIN are interrelated through a modular and structurally conserved intracellular tryptophan-rich domain

As shown in Table 2, hierarchical clustering supports the previously proposed link between the well-studied Plasmodium VSA gene families var (encoding PfEMP1 proteins), SICAvar, and surfin/pvstp1 [17]. Unexpectedly, the surfin/pvstp1 cluster includes two genes from $P$. 


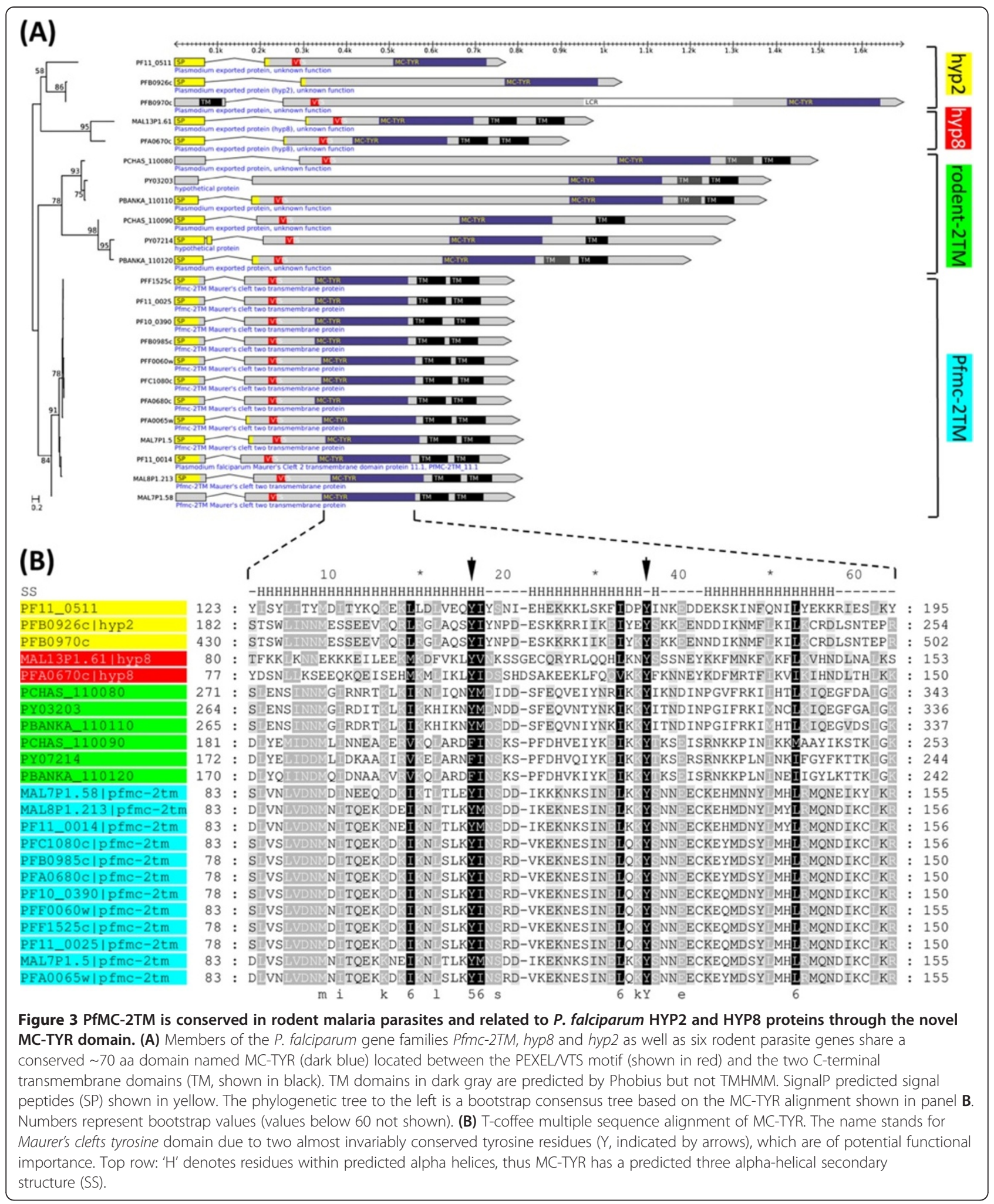

gallinaceum (which we named PgSurf1 and PgSurf2) that have very similar gene structures to $P$. falciparum surfin genes (Figure 4A), suggesting that SURFINs are conserved outside human malaria parasites. Further up the tree,
SURFIN/PvSTP1 proteins first group with $P$. knowlesi SICAvar proteins (28 genes and 214 pseudogenes, $\mathrm{MP}=$ $35.7 \%$, Avg.E $=0.013$ ) and then with another large cluster $(\mathrm{MP}=1.8 \%$; Avg.E $=1.9)$ containing the two P. falciparum 
gene families var (69 genes, 38 pseudogenes) and $d b l$ (4 genes) as well as six other DBL domain-containing proteins, including two MSP3 proteins (PF10_0348 and PF10_0355), MAEBL (PF11_0486), AMA1 (PF11_0344), giant protein Pf332 (PF11_0506), and one protein of unknown function (PFA0665w). Notably, still further up the tree the surfin/pvstp1/SICAvar/var gene cluster is joined by the large gene family pir (1,814 genes), uniting all major Plasmodium VSA gene families except rifin/stevor in a single gene cluster. However, with only 772 BLAST high-scoring segment pairs (HSPs) in total and an average E-value of 14 the connectivity between surfin/pvstp1/ SICAvar/var and pir was found to be extraordinarily low (Table 2). Because rifin/stevor genes are not part of this unified VSA gene cluster we conclude that there is no sequence-based evidence supporting a link of rifin/stevor and pir. However, as did others before us [20], we noticed suspicious similarities in some protein features (protein size, positioning of TM segments, secondary structural elements) that make such a link plausible (Additional file 4).

To better understand the nature of the relationship among SURFIN/PvSTP1, SICAvar, PfEMP1, and the DBLcontaining proteins, we investigated the sequence similarity matrix of this cluster as well as pairwise BLAST sequence alignments. As revealed by the sequence similarity heat map (Additional file 5), SICAvar and PfEMP1 proteins share no direct pairwise sequence similarity with each other but exhibit weak similarity with SURFIN/ PvSTP1 proteins (SICAvar: $\mathrm{MP}=36 \% / \mathrm{E}=0.013$; PfEMP1: $\mathrm{MP}=17 \% / \mathrm{E}=0.7)$. Notably, one $P$. knowlesi SICAvar protein (PKH_081360) and two of the DBL-containing proteins (Pf332 and PFA0665w) stand out by having high sequence similarity not only with paralogs of their respective gene families but also with SURFIN/PvSTP1 proteins $(\mathrm{PKH}$ 081360: $\mathrm{MP}=94 \% / \mathrm{E}=4 \mathrm{e}-13 ; \mathrm{Pf332}: \mathrm{MP}=89 \% / \mathrm{E}=$ 7e-6; PFA0665w: $\mathrm{MP}=89 \% / \mathrm{E}=0.2$ ). By examining the underlying BLAST alignments we found that BLAST consistently identified HSPs within the C-terminal intracellular regions of proteins, where, in SURFIN/PVSTP1 proteins, the HSPs overlapped with the previously described tryptophan-rich domain (WRD) [17]. In PfEMP1 proteins, the HSPs overlapped with the PfEMP1 intracellular region also known as the acidic terminal sequence (ATS) or VARC [15,39].

Subsequent multiple sequence alignments of SURFIN, PfEMP1 and SICAvar intracellular regions revealed a more complete picture of the relationship of these proteins than described previously [17]. We find that SURFIN WRD has a modular architecture consisting of two distinct and structurally conserved subdomains, which we named WRD-A and WRD-B (Figure 4). WRD-A and WRD-B occur in variable numbers and configurations in different gene families (Figure 4A). The typical WRD consist of one WRD-A (shown in green) and two WRD-B subdomains (shown in blue), which is found in P. falciparum (6-7 copies) and $P$. gallinaceum (4-5 copies) SURFIN proteins, the two fulllength $P$. vivax PvSTP1 proteins (single copy), and the two $P$. falciparum DBL-containing proteins, including Pf332 (single WRD copy, reported previously) and PFA0665w (1-2 WRD copies, new finding). Where WRDs occur in tandem, the first of the two WRDs is typically better conserved, as illustrated by the partially empty dashed rectangles representing cases where complete WRDs but not individual subdomains reached statistical significance in a HMMER3 search $(\mathrm{E} \leq 0.01)$. In other gene families, WRD is only partially conserved. The typical SICAvar protein contains a single WRD-A but no WRD-B subdomain, while PfEMP1 proteins carry one copy of both WRD-A and WRD-B separated by a $\sim 130$ aa long variable region. During our studies an experimentally determined threedimensional structure of the PfEMP1 intracellular ATS domain became available, which shows that ATS consists of a conserved core composed of four alpha helices [40] (Figure 4C). These four helices map nicely to the two conserved homology blocks corresponding to WRD-A and WRD-B (Figure 4B, red bars above alignments), supporting the biological significance of these alignments.

Notably, we identified at least one P. knowlesi SICAvar protein (SICAvar-like gene PKH_081360) with a completely conserved WRD (one WRD-A and two WRD-B), which explains the high BLAST sequence similarity of this protein with $P$. falciparum SURFINs and provides, for the first time, compelling alignment-based evidence that the two antigenically variant gene families var and SICAvar are evolutionarily linked via shared intracellular WRD domains. A TBLASTN search against the P. knowlesi genome using WRD-B of PKH_081360 as query identified one additional SICAvar antigen (PKH_102071, E=4e-11) carrying a WRD-B subdomain, but this gene appears to be severely truncated (295 aa) and lacks WRD-A, suggesting that PKH_102071 is possibly a pseudogene.

\section{New members of variant gene families predicted by sequence clustering}

Sequence clustering complements consensus-based strategies (using for example Pfam HMMs) for the identification of gene family members. We therefore examined gene clusters of sufficient quality $(J \geq 0.6$, Table 1$)$ for the presence of putative new gene family members. Table 3 lists newly predicted members of several $P$. falciparum gene families, including phist (4 genes), hyp4/hyp6 (3 genes), TSP_1 (2 genes), msp-7 (2 genes), msp-3 (1 gene), etramp (1 gene), hyp5 (1 gene), hyp15 (1 gene), and hyp10 (1 gene). In none of these cases could we identify obvious cases of misclassifications, suggesting that most of these genes are indeed potential novel members of these gene families.

To verify the credibility of these predictions, we examined the clustering result of the large and highly 


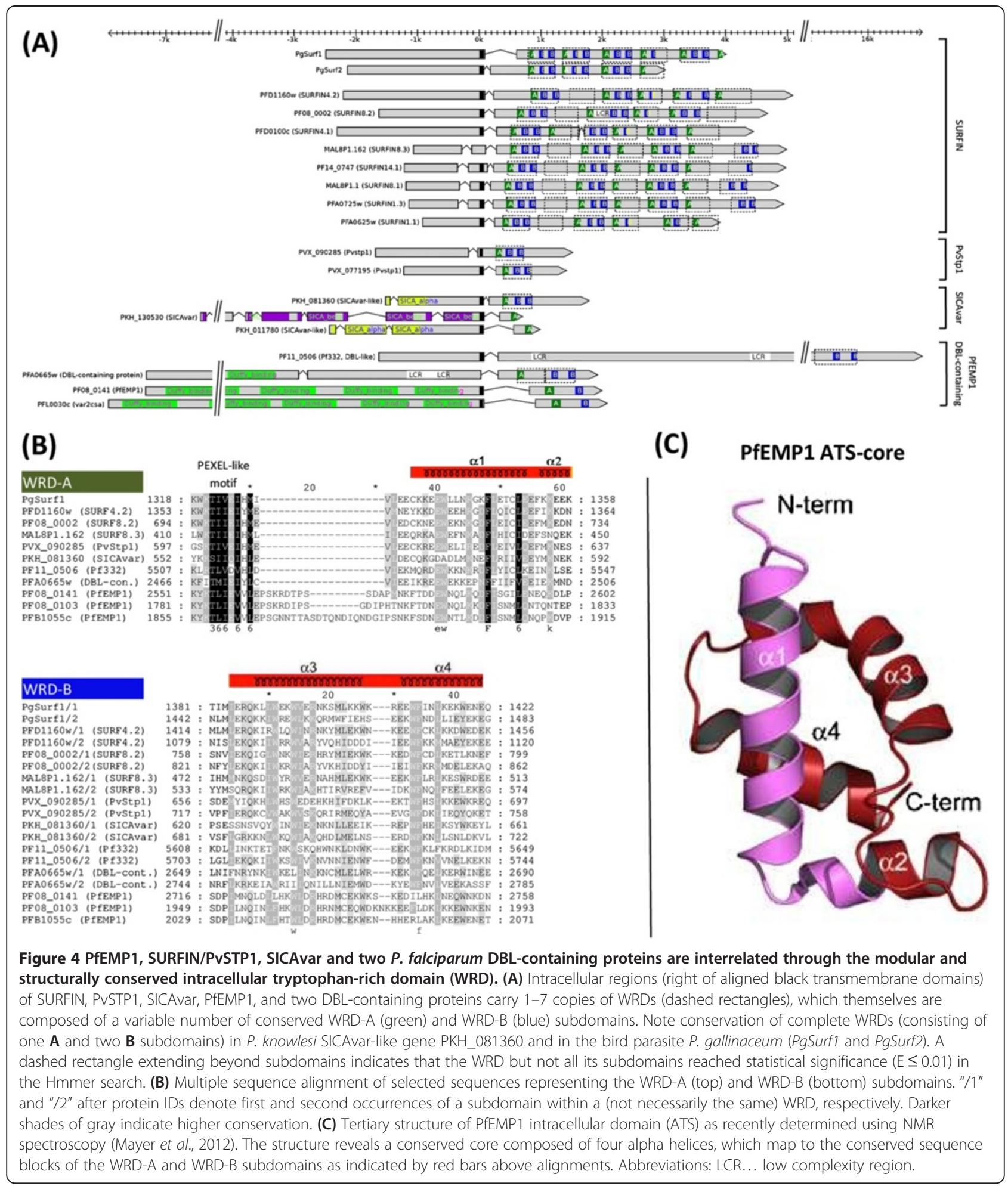

divergent phist gene family in more detail (Figure 5). Phist (Plasmodium helical interspersed subtelomeric) is a large subtelomeric gene family of exported proteins of unknown function with $\sim 70$ members in $P$. falciparum, $\sim 40$ members in $P$. vivax, and $\sim 27$ members in P. knowlesi. So far only one gene family member has been identified in each of the three rodent parasite genomes, suggesting extensive radiation of the phist gene family in primate parasites [23]. In P. falciparum, phist has been divided into three subfamilies (phist- $a$, phist- $b$, and phist-c). Another 
chromosome-internally expanded $P$. vivax gene family of 44 genes named rad (aka $p v-f a m-e$ ) has later been shown to be closely related to phist [10].

In our results, the phist- $a$, phist- $b$, phist-c, and rad gene families are resolved with high accuracy $(\mathrm{J} \geq 0.9)$ and as expected cluster together into the larger phist gene family ( $\mathrm{Sn}=0.97 ; \mathrm{Sp}=0.94 ; \mathrm{J}=0.92$ ) (Table 1 and Figure 5A). Phist-c first clusters with rad followed by phist- $b$ and phist- $a$, which differs from an earlier report suggesting that phist- $a$ and phist- $b$ form a clade with phist-c being the out-group [23]. In total, the phist cluster contains 22 full-length proteins that are currently not annotated as phist gene family members, including the four genes from P. falciparum mentioned earlier (PF14_0744, PF14_0745, MAL8P1.205 (GEXP13), and PF10_0015), two from P. vivax (PVX_003555 and PVX_096065), 14 from $P$. knowlesi (including four exported proteins from the $p k$ fam-e gene family that clusters with phist-b), and one from each P. berghei and P. chabaudi (PBANKA_070080 and PCHAS_093720). The two novel rodent parasite phist family members cluster with the phist-c subfamily and could be interesting targets for experimental studies of PHIST function in the rodent model system. Multiple protein sequence alignment of these putative new phist gene family members (Figure 5B) shows that key residues of the PHIST PRESAN domain (PF09687) are well conserved, suggesting that these 10 genes are likely true members of the phist gene family. Notably, the PHIST cluster also contains two proteins from P. gallinaceum, supporting previous findings that PHIST is conserved in the bird parasite [23]. We conclude that our clustering procedure resolves even challenging gene families correctly and is a successful strategy to identify putative new gene family members in P. falciparum and other Plasmodium species.

\section{Discussion}

In this study, we classified and investigated VSA and exported gene families across seven Plasmodium genomes. This led to several interesting findings, some of which may have application value.

\section{Conserved PIR proteins are targets for functional studies and vaccine development}

The identification of a single ultra-conserved PIR ortholog in each of the PIR-containing Plasmodium species (Figure 1) was highly unexpected given that pir is the largest and one of the most diverse gene families in Plasmodium parasites. Pir gene family sizes range from 68 in $P$. knowlesi to 838 in $P$. yoelii, with amino acid similarities ranging from $30-50 \%$ between $P$. chabaudi, P. yoelii and P. berghei and from $20-30 \%$ between $P$. chabaudi and P. vivax [41]. The same conserved PIR

Table 3 Putative new $P$. falciparum gene family members of variant gene families

\begin{tabular}{|c|c|c|c|c|c|c|}
\hline Gene family & New member & MP\# & MP \% & Avg. E-value & Avg. PID (Stddev) & Current annotation/comment \\
\hline \multirow[t]{4}{*}{ phist } & PF14_0744 & 21 & 9.9 & 0.2 & $27.6(5.6)$ & Exported protein, unknown function \\
\hline & PF14_0745 & 23 & 10.9 & 0.3 & $24.7(4.7)$ & Probable protein, unknown function \\
\hline & MAL8P1.205 & 40 & 18.9 & 0.0002 & 28.2(4.4) & GEXP13 \\
\hline & PF10_0015 & 88 & 41.5 & $7 e-14$ & $35.0(11.9)$ & Carries acyl-CoA binding domain \\
\hline \multirow[t]{3}{*}{ hyp4/hyp6 } & PFL2560c & 2 & 12.5 & $3 e-15$ & $46.3(14.4)$ & Probably truncated pseudogene \\
\hline & PFI0086w & 5 & 31.3 & 0.002 & $29.0(3.7)$ & Alternative start codon 40 aa downstream \\
\hline & PF14_0760 & 4 & 25.0 & 0.2 & $35.7(13.2)$ & Exported protein, unknown function \\
\hline \multirow[t]{2}{*}{ TSP_1 } & MAL8P1.45 & 48 & 23.3 & $7 e-17$ & $42.7(14.7)$ & Conserved protein, unknown function \\
\hline & PF08_0136b & 24 & 11.7 & $4 e-34$ & $35.3(16.3)$ & Von Willebrand factor A-domain related \\
\hline \multirow[t]{2}{*}{$m s p-7$} & PF13_0192 & 14 & 46.7 & 0.9 & $25.7(4.8)$ & Part of MSP7 gene cluster on chr13 \\
\hline & PF13_0194 & 3 & 10 & 3 & 28.8(3.3) & Part of MSP7 gene cluster on chr13 \\
\hline$m s p-3$ & PF10_0351 & 4 & 14.3 & 4 & $30.4(3.8)$ & Part of MSP gene cluster on chr10 \\
\hline etramp & PFL0065w & 2 & 2.8 & $9 e-15$ & $43.7(6.5)$ & Conserved protein, unknown function \\
\hline hyp5 & PF14_0743 & 10 & 100.0 & $3 e-5$ & $31.4(2.5)$ & Annotated hyp15 protein \\
\hline hyp 15 & PFB0950w & 3 & 100.0 & $3 e-18$ & $44.5(13.0)$ & Exported protein, unknown function \\
\hline hyp 10 & PF08_0001 & 1 & 50.0 & 3 & $34.6(0.0)$ & Exported protein, unknown function \\
\hline hyp 12 & MAL8P1.206 & 3 & 100.0 & $2 e-17$ & 47.1(4.9) & Exported protein, unknown function \\
\hline hyp 17 & PF14_0741 & 1 & 50.0 & $1 e-198$ & $91.2(0.0)$ & Split gene model; merged in PlasmoDB 9.0 \\
\hline
\end{tabular}

Clusters corresponding to variant gene families were searched for $P$. falciparum proteins that are currently not annotated as members of these gene families. Only clusters with a Jaccard index of at least 0.6 were considered in this search (Table 1). Annotated pseudogenes and gene fragment not shown. MP \# and MP\% indicate number and percentage of BLAST match pairs between the putative new member and all other proteins in the cluster representing that gene family. Avg. E-value and Avg. PID denote the average E-value and the average PID of these BLAST match pairs, respectively. ${ }^{\mathrm{a}}$ Unusual phist gene family member carrying an $\mathrm{N}$-terminal acyl-CoA-binding protein domain (Pfam PF00887). 

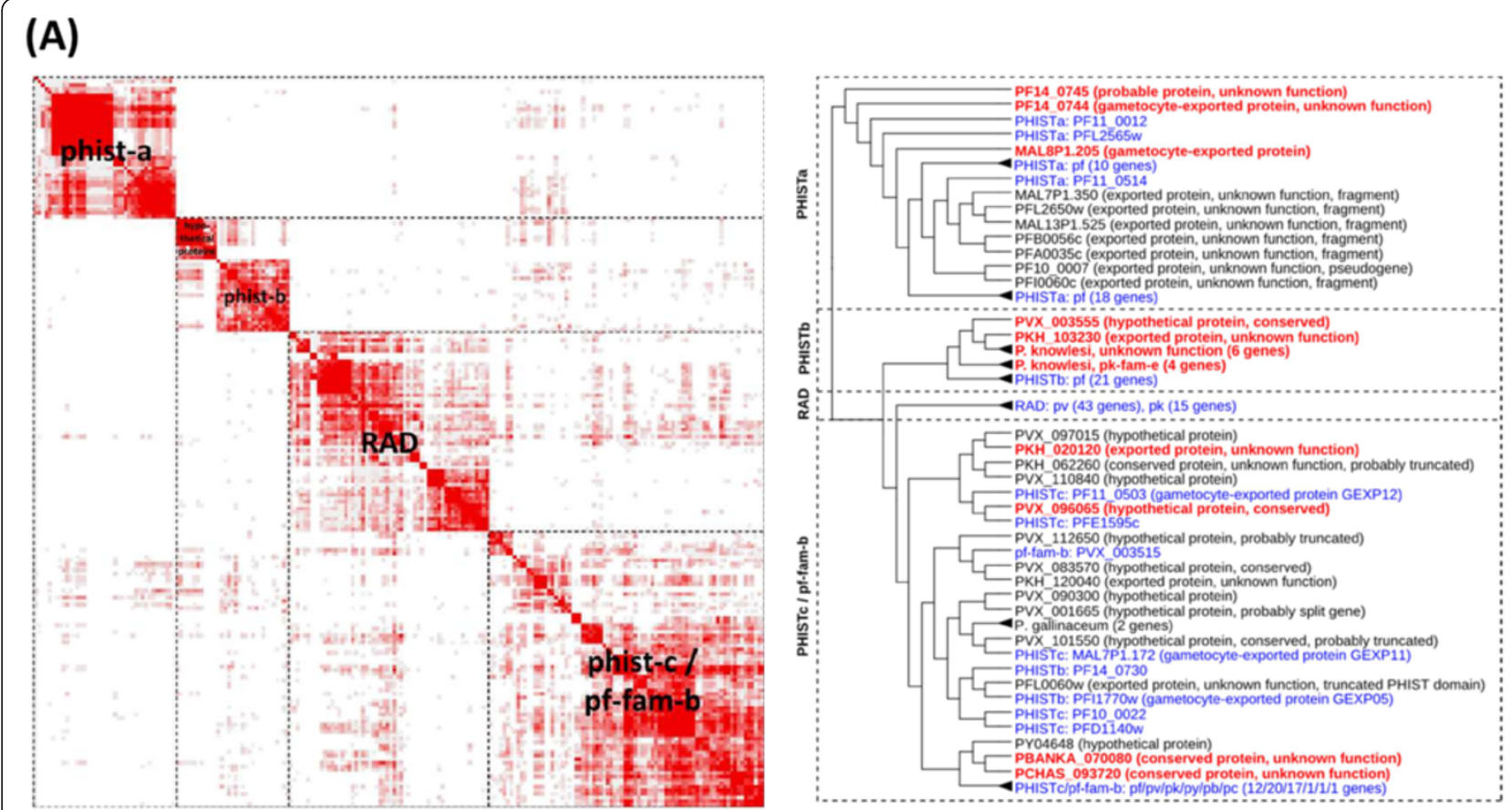

\section{(B)}

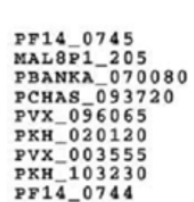

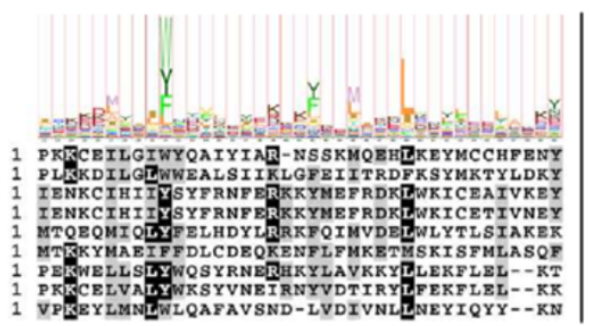
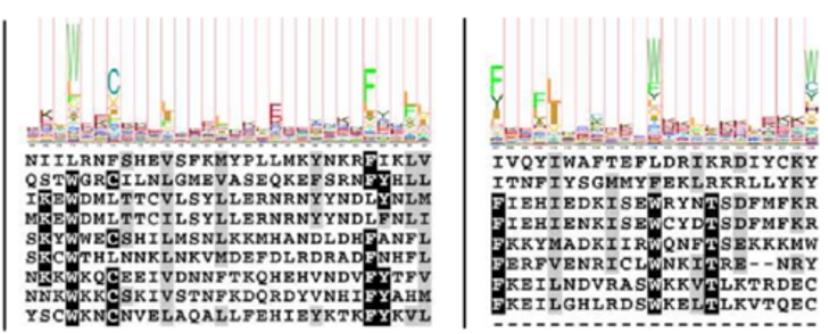

Figure 5 Predicted new members of the exported gene family phist in several Plasmodium species, including rodent parasites. (A) Left: Heat map of pairwise BLAST sequence similarities between all proteins of the PHIST cluster. Shades of red indicate degree of similarity (red = high similarity, white $=$ no similarity). Right: Compressed PHIST dendrogram extracted from the hierarchical tree. Annotated phist gene family members indicated in blue. Putative new gene family members shown in red (full-length proteins) or black (annotated pseudogenes or gene fragments). Note the putative new phist gene family members in rodent parasites at the bottom. Black triangles indicate collapsed subtrees with numbers of genes contained in this subtree specified in parentheses. (B) Top: Pfam family PF09687 (PRESAN domain) sequence logo highlighting conserved key residues of the phist gene family. Bottom: Corresponding conserved blocks extracted from a T-coffee multiple sequence alignment of selected putative new PHIST members. Note that these putative new gene family members have most key residues of the PRESAN domain conserved, suggesting that they are likely true members of the diverse phist gene family.

orthologs were found recently in an independent work studying genetic diversity of the global $P$. vivax population [42]. It was shown that $P$. vivax gene PVX_113230 (one of the five conserved PIR orthologs) is completely invariant across four strains sampled from different sites around the world and also exhibits conserved synteny in rodent malaria parasites. The fact that these highly conserved PIR orthologs could be identified in our study clearly highlights the value of comparative genomics in general and of comparative gene family classification in particular.

The discovery of ultra-conserved PIR orthologs has two important implications. Firstly, these genes can now serve as starting points to elucidate PIR function, which is still unknown. For example, gene knockouts of the conserved PIR orthologs in the more accessible rodent model system may result in important and measurable phenotypes. Alternatively, tagging the conserved PIR orthologs with different fluorescence markers coupled with the manipulation of their expression levels should also help clarifying PIR localization and function. PVX_113230 has a distinct expression profile in erythrocytes relative to most other vir genes [43] and therefore may serve a different, ancestral function. If true, insights from the proposed experiments may not be directly applicable to other members of the gene family, but should nevertheless be informative.

Secondly, the finding of a conserved PIR ortholog in $P$. vivax has potential ramifications for $P$. vivax vaccine development. If, like other VIR proteins [19], PVX_113230 is confirmed as surface-exposed antigen, antibodies 
raised either against PVX_113230 or its hypothetical host receptor(s) could elicit exquisite cross-strain immunity. That parasite genes with limited genetic diversity can be effective vaccine targets was recently demonstrated in P. falciparum, where antibodies against an essential and highly conserved ligand for erythrocyte invasion (PfRh5) elicited exquisite neutralizing cross-strain immunity $[44,45]$. This seemingly paradoxical situation, that a highly conserved parasite protein can be successfully targeted by the host immune system, makes sense if one imagines PfRh5 as functionally important and therefore conserved component of a host-parasite interaction complex. Within such a complex, PfRh5 would not be under direct immune attack because it is efficiently shielded by immunodominant paralogs that act as red herrings or "smokescreen" [46]. A similar model has been proposed for rif/ stevor and surfin genes in the context of the merozoite invasion process [47]. If this model applies to PIR proteins, then PVX_113230 could be a promising target for the development of a $P$. vivax vaccine.

\section{Lipid-binding PYST-A proteins potentially involved in cholesterol salvage}

Another family of surface antigens that is currently without known function is the large rodent parasite gene family pyst-a (named $p c-f a m-1$ and $p b-f a m-1$ in $P$. chabaudi and $P$. berghei, respectively). Studies of pyst-a expression have shown that the pyst- $a$ family member and glutamaterich protein Pc90 (also known as Pc(em)93, Pc(em)96, and Pch105/RESA [48]) is the immunodominant protein within red blood cell ( $\mathrm{RBC}$ ) membranes and localizes to the cytoplasmic face of the membrane [49,50]. More recently, RBC membrane localization of a pyst-a homolog (PBANKA_083680) has also been demonstrated in $P$. berghei [51]. Pyst-a genes are concurrently expressed in large numbers without evidence of differential expression in response to different host environments [52], suggesting that altering expression of pyst-a gene family members is not an immune evasion strategy of the parasite. Using bioinformatics sequence and structural analysis we predict the existence of a StAR-related lipid-transfer (START) domain in pyst-a proteins (Figure 2). START is the archetypical domain found in the steroidogenic acute regulatory (StAR) protein, which is part of a multi-protein complex termed the "transduceosome" whose function in mammals is to transfer cholesterol across the two mitochondrial membranes and to initiate the first enzymatic step in steroid biosynthesis [53,54]. Cholesterol is also a major lipid fraction in the membrane bilipid layer and is required for membrane genesis. Plasmodium parasites cannot synthesize cholesterol de novo [55]. It has been shown that host cell membranes of $P$. falciparum-infected erythrocytes show a 50\% decrease in the cholesterol/ phospholipid ratio compared to uninfected cells [56]. In addition, malaria infections result in hypocholesterolaemic conditions [57]. Together these observations suggest that the parasite scavenges required cholesterol from both host erythrocyte membranes and plasma. How this is accomplished by the parasite is currently not understood. Based on the presence of a cholesterol-binding START domain in RBC membrane-localized PYST-A proteins we hypothesize that PYST-A is part of a molecular machinery that diverts cholesterol away from erythrocyte membranes to fuel parasite growth within erythrocytes. Notably, $P$. chabaudi PcEMA1 proteins could constitute a second component of this proposed cholesterol import machinery. We found five PC-FAM-1 proteins (PCHAS_060180, PCHAS_140150, PCHAS_137050, PCHAS_110050, and PCHAS_042050) that share sequence similarity with both PYST-A (C-terminal) and PcEMA1 proteins (N-terminal). We could not find obvious problems with the gene models of these five proteins, suggesting that they are genuine PYST-A/PcEMA1 protein hybrids. Co-occurrence of PYST-A and PCEMA1 domains within the same polypeptide suggests physical interaction between PYST-A and PcEMA1 proteins. This possibility is supported by the fact that PcEMA1 proteins also localize to the cytoplasmic face of the RBC membrane [58]. It remains to be shown why rodent malaria parasites maintain so many PYST-A proteins compared to primate malaria parasites. PYST-A proteins are probably immunogenic in rodent but not primate parasites, putting PYST-A proteins under strong selective pressure to diversify. Alternatively, massive pyst-a gene amplification could increase the efficiency of cholesterol salvage, probably satisfying an elevated need for cholesterol in rodent parasites or compensating for lower cholesterol levels in rodent blood.

\section{Pfmc-2TM - conserved beyond $P$. falciparum}

Another interesting erythrocyte surface-expressed gene family of currently unknown function is the $P$. falciparum gene family Pfmc-2TM. The 12 members of the Pfmc-2TM gene family encode putative functional and highly similar (avg. global pairwise PID $=76 \%$, range 61 $96 \%$ ) basic membrane proteins that, based on structural features, are grouped within the larger 2TM superfamily. The 2TM superfamily consists of 200-300 aa long proteins that on their first exon carry a signal peptide followed by a N-terminal PEXEL motif and two Cterminal TM domains on their second exon. Besides Pfmc-2TM, the 2TM superfamily includes rif/stevor as well as genes in P. knowlesi, P. vivax, and the rodent parasite genomes that share similar architectural features [59]. It is important to note that, other than pir, the large 2TM superfamily is currently united solely based on gene structural features and not sequence similarity; in fact, to date no PfMC-2TM sequence homologs have 
been identified in $P$. falciparum or any other species. PfMC-2TM proteins have been localized to the Maurer's clefts $[18,36]$, the parasitophorous vacuole, parasitophorous vacuole membrane [36], and the erythrocyte surface [60]. At this latter location, 2TM proteins including PfMC-2TM are thought to interact with the host immune system through a surface-exposed and hypervariable loop region that is located between the two TM domains [60]. PfMC-2TM proteins are most highly expressed in the mid-trophozoite stage and have been shown to undergo expression switching, suggesting that the Pfmc-2TM gene family plays a role in antigenic variation $[18,35]$.

We provide first evidence that the Pfmc-2TM gene family has additional members both within and outside the $P$. falciparum species (Figure 3). Experimental clues for the existence of potential Pfmc-2TM homologs in the rodent Plasmodium genome already existed, but the identity of these genes remained unknown [36]. Furthermore, we showed that PfMC-2TM proteins contain a conserved and putative functional domain located between PEXEL and the two TM domains, which we named MC-TYR. These two findings have important implications for future studies of PfMC-2TM function. Experimental doubleknockout studies can now be attempted in rodent parasites on the two Pfmc-2TM gene family members to see if they produce an observable phenotype. Given the reduced number of Pfmc-2TM gene family members in rodent malaria parasites compared to $P$. falciparum and the more tractable rodent model system, such studies should now be feasible. Two additional observations suggest that hyp2, hyp 8 , and the six rodent malaria proteins are genuine members of the extended Pfmc-2TM gene family. First, all but three of the putative new Pfmc-2TM members (PCHAS_110090, PFB0926c, and PFB0970c) have a similar basic isoelectric point (9.2-10.5 pH) as known PfMC$2 \mathrm{TM}$ proteins. Second, all newly identified members that have two TM domains carry a single proline residue within the second TM domain, another known characteristic of PfMC-2TM proteins [18]. Proline residues internal to helices are often found in transporters, channels, and receptors, and tend to be conserved between homologous proteins. In contrast, we did not observe conservation of the two cysteine residues preceding the TM domains [18]. Also the fact that no TM domains are present in three of the proteins carrying MC-TYR is intriguing and suggests that the presence of two TM domains is not essential for a possible MC-TYR function. It is also noteworthy that most of the hyp 2 and hyp 8 gene family members are more broadly expressed throughout the intra-erythrocytic life cycle than PfMC-2TM proteins, showing expression not only in the mid-trophozoite stage but also in early ring and schizont stages (PFB0926c, PFB0970c, MAL13P1.61, and PFA0670c) as well as in gametocytes (hyp8) (PlasmoDB 9.0).
This suggests that the function of MC-TYR containing proteins is not restricted to trophozoites. Our clustering results do not provide evidence for a link between Pfmc-2TM and the pyst- $b$ gene family as has been proposed previously based on gene structural features [18].

\section{WRD as missing link between major Plasmodium VSA gene families}

Besides the large 2TM superfamily, malaria parasites express additional VSA gene families on cell surfaces. Among them are the var gene family in $P$. falciparum and SICAvar in P. knowlesi, both of which encode proteins expressed at the erythrocyte surface and are known to undergo antigenic variation. SURFIN, a family of $10 \mathrm{P}$. falciparum proteins, has been shown to be expressed on the surfaces of both infected erythrocytes and merozoites [17]. By examining intracellular regions of co-clustered SURFINs, PfEMP1 and SICAvar proteins, we now better understand the sequence conservation pattern present among these major Plasmodium VSAs (Figure 4). We found that SURFIN proteins contain twice as many WRDs as reported previously [17] and that each WRD consists of three conserved blocks. The first block, named WRD-A (40-60 aa), is found once in each of the WRDs, whereas block two and three represent two copies of a second subdomain that we named WRD-B (40-50 aa). Winter et al. previously described the conservation pattern among WRD-containing proteins in terms of shared conserved elements denoted S1, S2, S2*, S2**, and PEXEL-like motif [17]. Compared to this earlier study, we find that WRD-A corresponds to segment S1 plus five additional C-terminal amino acids in WRD-A, which map to the second alpha helix of the protein structure (Figure 4B). WRD-B corresponds roughly to the C-terminal half of segment $\mathrm{S} 2$, and segment S2* (featuring a duplication within antigen Pf332) is in fact the first of the two WRD-B copies found within this protein. The short $\mathrm{S} 2 *$ subsegment, indicating another duplication at the end of each WRD in SURFIN 4.2, is in fact the most conserved part of the second copy of the WRD-B subdomain in this protein. The PEXEL-like motif localized within the S1 segment was also found to be well conserved in our study (Figure 4B). Interestingly, this PEXEL-like motif currently has no correspondence in the reported tertiary structure (Figure $4 \mathrm{~B}$ and Figure $4 \mathrm{C}$ ), which might be explained by the fact that the ATS-Core deletion construct that was used to determine the ATS structure lacked this PEXEL-like motif [40]. It thus remains to be shown if the PEXEL-like motif is in fact part of ATS-Core or serves another conserved function in WRDs.

Importantly, our data provides the first conclusive alignment-based evidence that the two major antigenically variant gene families in $P$. falciparum (var) and in $P$. knowlesi (SICAvar) share common evolutionary origin, 
substantiating the usefulness of the $P$. knowlesi-rhesus monkey model for the in vivo study of $P$. falciparum antigenic variation. In 2005, Winter et al. provided the first bioinformatics evidence that PfEMP1 and SICAvar proteins are probably evolutionarily linked through their intracellular domains, which share partial sequence similarity with the intracellular tryptophan-rich domain (WRD) of SURFIN proteins [17]. Short conserved sequence motifs have also been identified in var and SICAvar introns [11] as well as in extracellular regions of these proteins [61]. However, at present the picture remains highly fragmented and reported sequence similarity of intracellular regions between PfEMP1 and SICAvar is essentially limited to a highly conserved six amino acid long PEXEL-like motif, questioning the robustness of these observations. Our study provides new evidence for common ancestry of PfEMP1 and SICAvar, which rests on three critical observations. Firstly, not only WRD-B (formerly segment S2) but also WRD-A (formerly segment S1) is entirely conserved in PfEMP1 proteins. Secondly, at least one SICAvar protein (PKH_081360) has a completely conserved WRD, consisting of one WRD-A and two WRD-B subdomains. Thirdly, complete WRDs are conserved in at least two other P. falciparum proteins (Pf332 and PFA0665w), which are clearly related to PfEMP1 proteins through $\mathrm{N}$-terminal DBL domains. Why were these sequence similarities not detected previously? Unlike SURFINs, PfEMP1 proteins have WRD-A and WRD-B separated by a larger $(\sim 130$ aa) variable insertion, which in the protein structure does not adopt a stable fold [40]. Similarly, compared to SURFINs, PfEMP1 proteins carry additional insertions both within WRD-A ( 20 aa insertion) and WRD-B ( $\sim 3$ aa insertion) (Figure $4 B)$. In WRD-A, this insertion splits the highly conserved PEXEL-like motif from the rest of the WRD-A subdomain (Figure 4B). The presence of these insertions in PfEMP1 proteins combined with the fact that WRD-B is absent in all but one SICAvar protein likely thwarted earlier attempts to find significant sequence conservation between PfEMP1 and SICAvar proteins $[17,20]$. It is not too surprising that larger blocks of conserved sequence between PfEMP1 and SICAvar are confined to intracellular regions as extracellular regions are likely under strong diversifying selection pressure from the host immune system. Consistent with earlier reports, we did not find evidence that WRD or any of its subdomains is conserved within the large pir gene superfamily of Plasmodium surface antigens [17].

\section{What is the function of WRD?}

Despite this now much improved picture of the conservation structure of PfEMP1, SICAvar, and SURFIN intracellular domains, many fundamental questions remain. Why do SURFINs have multiple copies of complete WRDs while most other gene families carry only a single WRD? What is the functional significance of having two WRD-B subdomains (SURFINs and Pf332) vs. having only one WRD-B subdomain (PfEMP1)? Why is WRD-B found in only a single SICAvar protein (PKH_081360) while all other members of the SICAvar gene family carry only the WRDA subdomain? Unfortunately, the molecular function of WRD is currently not well understood, so these questions are difficult to answer. Studies have shown that ATS of PfEMP1 interacts either directly or indirectly via the knob-associated histidine-rich protein (KAHRP) with erythrocyte membrane skeletal proteins, including actin and spectrin [39,62-65]. ATS is thus believed to anchor PfEMP1 at the membrane of infected RBCs, which strengthens and stabilizes the roots of PfEMP1-endothelial receptor interaction. Similarly, Pf332, which contains a single complete WRD near its C-terminal end, was found to contribute to the overall deformability of $P$. falciparuminfected erythrocytes through anchoring and scaffolding $[66,67]$, probably through WRD-mediated binding of Pf332 to actin [68]. It seems therefore plausible that the main function of WRD is to provide a scaffold for anchoring surface antigens like PfEMP1 to the RBC cytoskeleton. The function of having many WRDs resulting from gene family expansion and/or intraprotein domain amplification is then probably to increase the strength of this scaffold. This would explain why particularly $P$. falciparum, which compared to other malaria parasites has strong cytoadherence properties, expresses so many WRDs in form of the surfin gene family. SURFINs have not yet been implicated in anchoring surface antigens to the erythrocyte cytoskeleton, but we think that this is a plausible hypothesis that should be tested experimentally. It is also worth mentioning that the ABBABBABB-like repeat structure of the SURFIN WRDs is reminiscent of similar repeats present in other cytoskeleton-binding proteins, including the vertebrate titin and the invertebrate twitchin muscle proteins [69]. Regardless of the exact function of WRD, its modular nature suggests that different proteins carrying distinct WRD subdomains act in concert to perform this function. P. knowlesi protein PKH_081360, which appears to be the only functional protein in the $P$. knowlesi genome carrying both WRD-A and WRD-B, thus probably interacts physically with other, WRD-A-containing SICAvar proteins to anchor SICAvar to the cytoskeleton. With the refined domain structure presented here it should now be possible to test this prediction experimentally. Ultimately, such experiments will hopefully expand our knowledge of how $P$. falciparum accomplishes cytoadherence at the molecular level, which is responsible for much of the virulence of this parasite. 


\section{Conclusions}

Sensitive clustering of protein sequences followed by manual examination of clustering results revealed many new insights into variant surface antigens of malaria parasites, including atypical and new gene family members, novel domains, new protein function predictions, and a better understanding of the evolutionary relationship and origin of these important proteins. The findings presented in this work can now jump-start follow-up experimental research around the world, most importantly to elucidate the function of PVX_113230 and to explore its potential value as $P$. vivax vaccine target.

\section{Methods}

\section{Protein sequence data set}

The raw protein sequence data set included 259,778 protein sequences from 18 species, including seven Plasmodium species ( $P$. falciparum, $P$. vivax, $P$. knowlesi, $P$. yoelii, $P$. berghei, $P$. chabaudi, $P$. gallinaceum), three related Apicomplexan parasites (Theileria parva, Toxoplasma gondii, Cryptosporidium parvum), seven other, well-studied model organisms (Drosophila melanogaster, Caenorhabditis elegans, Monosiga brevicollis, Saccharomyces cerevisiae, Arabidopsis thaliana, Chlamydomonas reinhardtii, Escherichia coli), and human. Protein sequences were obtained from various sources as summarized in Additional file 6. The rationale for including additional species besides Plasmodium was that on the one hand we hoped to improve the sensitivity of the clustering algorithm through the transitivity principle and that on the other hand we expected that functional insights could be gleaned from functionally characterized nonPlasmodium proteins that cluster with uncharacterized Plasmodium proteins.

\section{P. gallinaceum gene prediction}

Because gene annotations for $P$. gallinaceum were not available when this study was initiated, $P$. gallinaceum protein-coding genes were predicted using our own homology-based gene predictor genBlastG (version 1.28) [70]. P. gallinaceum supercontigs $(4,996)$ were obtained from the Sanger FTP site (http://sanger.ac.uk/ pub/pathogens/Plasmodium/gallinaceum/) and annotated with genBlastG using protein sequences from the well-curated P. falciparum gene set (PlasmoDB 6.0) as query (parameters: -c 0.5 -s 0 -r 1 -gff -pro -b). This resulted in 3,141 predicted $P$. gallinaceum proteincoding gene models. It is important to note that this $P$. gallinaceum gene set was created solely for the purpose of comparative gene family classification and should be considered highly preliminary. While $P$. gallinaceum protein-coding genes with sufficient sequence similarity to $P$. falciparum proteins are expected to be well represented, genes or exons with low similarity might be absent or mispredicted. Also, the currently low sequencing coverage of the P. gallinaceum genome (three-fold) means that many gene models will be missing.

\section{Gene model improvement}

Besides predicting gene models for P. gallinaceum, we also improved existing gene model annotations for the genomes of $P$. vivax, $P$. knowlesi, $P$. chabaudi, $P$. berghei, and $P$. yoelii using a previously developed homologybased gene model improvement pipeline [12,71]. Briefly, protein sequences of all protein-coding $P$. falciparum genes were used as query to run both genBlastG [70] and GeneWise [72] against the other Plasmodium genomes. To ensure the quality of predicted gene models, only predictions that encoded for protein sequences with high global sequence identity (PID $>=60$ ) with the query gene were kept. If multiple predictions overlapped by more than $5 \%$ of their coding exons, only the prediction with the highest PID to the query was kept (filtration step). In a subsequent merging step, predicted and existing gene models were merged into a hybrid gene set, retaining predictions that (a) did not overlap with existing gene models or (b) showed a PID improvement of at least 5\% over overlapping existing gene models. As in the filtration step, existing and predicted gene modes were considered as overlapping if more than $5 \%$ of their coding exons overlapped. This hybrid gene set then served as the basis for the subsequent protein sequence clustering step.

\section{Hierarchical clustering}

The raw set of 259,778 protein sequences was filtered to retain only longest isoforms, which reduced the number of protein sequences to 171,476 . Low-complexity regions were masked with TANTAN [73] (parameters: $-\mathrm{p}-\mathrm{s}$ $0.99-\mathrm{r}$ 0.005) before BLAST analysis because lowcomplexity filtering as performed by BLAST was found to be insufficient to mask more complex repeats, causing clustering of non-homologous proteins. NCBI BLASTP version 2.2.25+ (http://blast.ncbi.nlm.nih.gov) (parameters: -evalue 100 -num_descriptions 2000 -outfmt 6 -word_size 2 -lcase_masking) was then used to compare protein sequences in an all- $v s$.-all pairwise manner, resulting in a total of 24,206,683 HSPs. HSPs were then filtered to retain only the best HSP (= lowest E-value) per protein sequence pair, resulting in 12,224,106 symmetrified best HSPs. E-values were transformed into positive dissimilarity values for hierarchical clustering using the formula $200-\min \left(200,-\log _{10}(\mathrm{E}\right.$-value/100)), whereas Evalues of 0 were assigned the minimum dissimilarity value of 0 . Hierarchical clustering was performed using MCUPGMA (version 1.0.0) [28] obtained from http://www. protonet.cs.huji.ac.il/mcupgma/ (parameters: -max_distance $=200$-iterations $=100$ ). 


\section{Cluster extraction}

Cluster extraction from the hierarchical tree was performed using custom Perl scripts (Figure 6). Starting at a leaf node corresponding to a known gene family member, we moved up the hierarchical tree until specificity $(=\mathrm{TP} /(\mathrm{TP}+\mathrm{FP}))$ dropped below 0.1 or sensitivity $(=\mathrm{TP} /$ $(\mathrm{TP}+\mathrm{FN})$ ) reached the maximum value of 1.0 (i.e. all known family members are contained within this subtree). For each internal node visited during this bottom-up tree traversal we computed and noted its Jaccard index $(=\mathrm{TP} /$ $(\mathrm{TP}+\mathrm{FP}+\mathrm{FN}))$. The above procedure was repeated for each known member of a gene family. Finally, the internal node with the highest Jaccard index was returned as representative gene cluster for this gene family. Annotated pseudogenes or proteins of non-reference species were not considered when computing specificity, sensitivity, and Jaccard indices. Defined reference species for each gene family are shown in Table 1 and correspond to species that have gene numbers shown in parentheses.

\section{PIR conservation analysis}

For each of the five pir subfamilies (bir, cir, yir, kir, vir), all members of a subfamily were globally aligned to all members of the other four subfamilies. Global protein sequence alignment was performed with ClustalW (version 1.83)
[74] using the default BLOSUM matrix and default parameters. The distribution of global percent identity (PID) values shown in Figure $1 \mathrm{~B}$ was generated using the 'boxplot' function of $\mathrm{R}$ with the range parameter set to 2.5. The multiple sequence alignment shown in Figure 1A was computed online at http://tcoffee.crg.cat using T-Coffee with default parameters [75]. The alignment was then pruned and formatted with GeneDoc 2.7 [76].

\section{Pyst-a function and structure prediction}

Domain annotations shown in Figure 2A were generated with HMMER3 [77] (http://hmmer.janelia.org/) using HMMs downloaded from SUPERFAMILY version 1.75 [78] (http://supfam.cs.bris.ac.uk/SUPERFAMILY/downloads.html\#Models) and visualized with FeatureStack [79]. Downloaded HMMs were first converted into a HMM database using hmmpress (default parameters) and then searched against Plasmodium protein sequences using hmmscan (default parameters). The protein structure prediction of PF14_0604 was generated using I-TASSER server [80,81] with default parameters. TM-score, RMSD, and PID correspond to values outputted by I-TASSER, which used TM-align to compute these values [82]. Protein sequence alignment and secondary structure prediction were also obtained from the

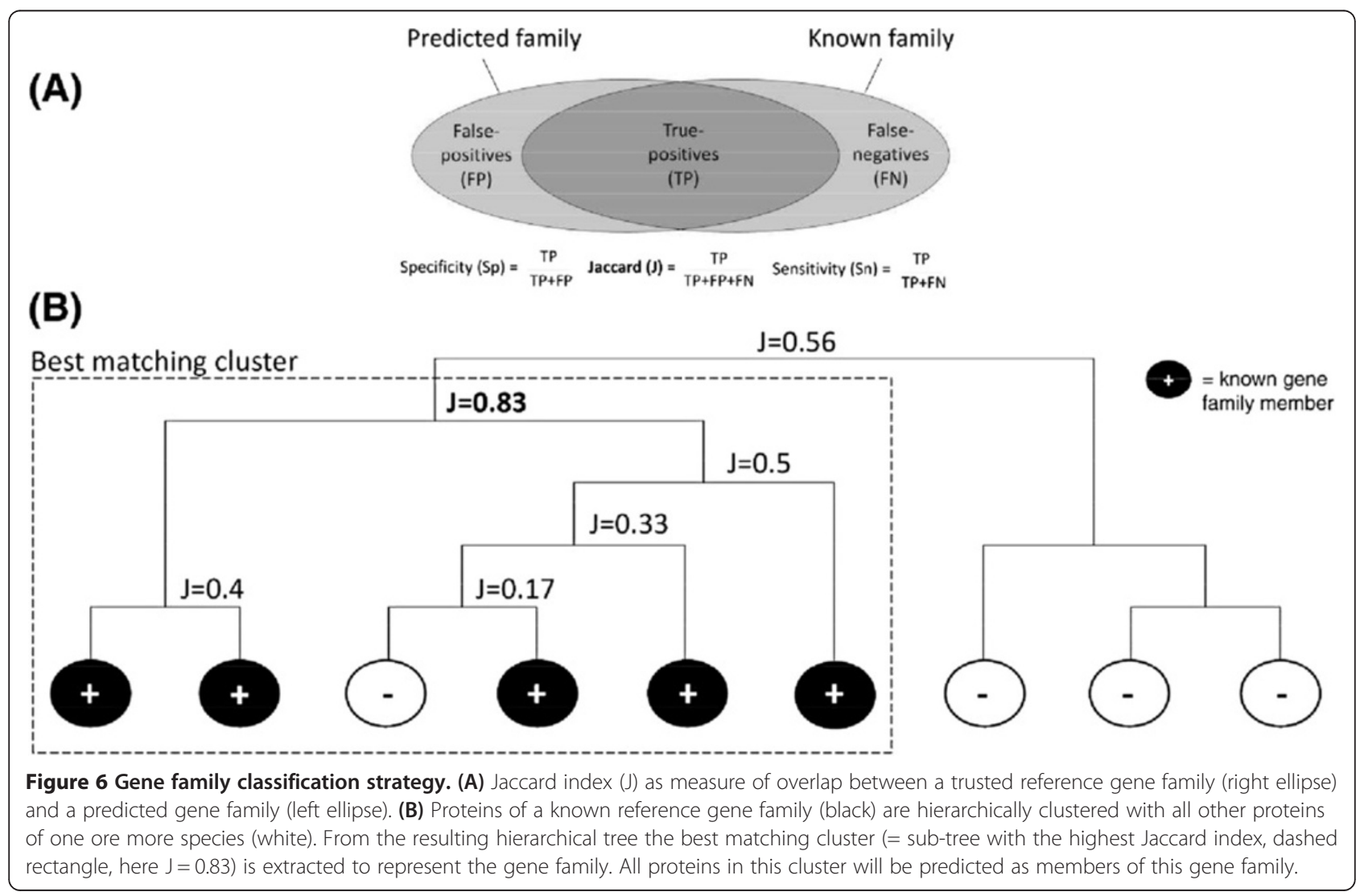


I-TASSER output. Both predicted and template protein structures were rendered with PyMOL (The PyMOL Molecular Graphics System, Version 1.3, Schrödinger, LLC).

\section{Identification and phylogenetic analysis of MC-TYR} PfMC-2TM, HYP2 and HYP8 protein sequences clustering together in the hierarchical tree (Figure 4) were aligned using the T-Coffee web service [75]. The MCTYR domain was identified by manual inspection of the resulting multiple sequence alignment. Transmembrane domains were predicted using the TMHMM standalone version (v2.0c) [83] and the EBI Phobius Web server [37] (http://www.ebi.ac.uk/Tools/pfa/phobius). Coloring of the MC-TYR multiple sequence alignment was performed with GeneDoc [76] using the 'Shade 4 Levels' option. The secondary structure of MC-TYR was predicted with Jpred 3 [84] using the multiple sequence alignment of Figure 4B as input. The phylogenetic tree shown in Figure 3 was produced with MEGA5 [85] using maximum likelihood and 100 bootstrap iterations.

\section{Identification of WRD-A and WRD-B subdomains}

Guided by local BLAST sequence similarities we compiled a hand-curated set of partial SURFIN, PvSTP1, SICAvar, PfEMP1, and DBL-containing protein sequences. These sequences were aligned using PSI-Coffee from the T-Coffee web site [75] with default parameters. Resulting multiple sequence alignments were manually curated with GeneDoc 2.7 [76] and poorly aligned sequences were removed. $\mathrm{Cu}$ rated multiple sequence alignments representing WRD-A (Additional file 7, 19 sequences) and WRD-B (Additional file 8,32 sequences) subdomains were then converted into Stockholm format at http://myhits.isb-sib.ch/cgi-bin/reformat and used as input for HMMER3 searches (default parameters). We also hand-curated a multiple sequence alignment representing the complete WRD consisting of one WRD-A and two WRD-B subdomains (Additional file 9). HMMER3 predictions were then visualized on top of gene models (Figure 4A) using FeatureStack [79]. Only matches with an E-value of 0.01 or lower are shown. TM domains were identified with TMHMM (v2.0c) [83]. Domain matches in intracellular regions of proteins shown in Figure 4A were also identified with HMMER3 using Pfam v26.0 domains as input (E-Value $\leq 1 \mathrm{e}-10)$ [86]. Annotated domains correspond to Pfam entries PF12887 (SICA_alpha), PF12878 (SICA_beta), and PF05424 (Duffy_binding). Gene structures of PgSurf1 and PgSurf2 were manually curated based on the presence of two large open reading frames in both genes, representing exon 1 and exon 2. Predicted protein sequences of PgSURF1 and PgSURF2 are provided in Additional file 10.

\section{Data access}

We set up a project Web page (http://genome.sfu.ca/projects/gfc-plasmodium) providing clustering results for all Plasmodium gene families examined in this study. For each gene family, this data includes: cluster dendrogram (Newick format); sequence similarity matrix (tab-delimited format); list of protein accessions and descriptions (tab-delimited format); protein sequences (multi-fasta format); and images of annotated gene models in form of both a static and an interactive HTML page. These images also contain cluster dendrograms and intraerythrocytic expression profiles for $P$. falciparum and $P$. vivax proteins.

\section{Additional files}

\begin{abstract}
Additional file 1: Phylogenetic tree of selected malaria parasite species. Symbols next to species names indicate infected host species, including human, monkeys, rodents, and birds. The phylogenetic tree is reproduced from [87] and inferred from partial mitochondrial genomes (5,580 bp). Numbers above branch points represent posterior probabilities in percent, and the scale bar represents the number of nucleotide substitutions per site. The avian Haemosporida Leucocytozoon sabrazesi was used as out-group.
\end{abstract}

Additional file 2: Extended version of Table 1 with gene numbers from additional species.

Additional file 3: Subtree of the vir gene family classification result, highlighting gene models and placement of the conserved pir orthologs.

Additional file 4: Similarities of protein secondary structural features between two PIR and RIFIN proteins.

Additional file 5: Sequence similarity heat-map of all SURFIN, PfEMP1, and SICAvar proteins, pointing out atypical gene family members with high cross-family similarity. Additional file 6 lists all species and data sources of the proteins classified in this study.

Additional file 6: Species and data sources of the proteins classified in this study.

Additional file 7: Multiple sequence alignment underlying the WRD-A domain.

Additional file 8: Multiple sequence alignment underlying the WRD-B domain.

Additional file 9: Multiple sequence alignment underlying the complete WRD domain.

Additional file 10: Protein sequences of the two newly discovered SURFIN proteins in P. gallinaceum (PgSURF1 and PgSURF2).

\section{Competing interests}

The authors declare that they have no competing interest.

\section{Authors' contributions}

NC and CF conceived the study. CF carried out data analysis and interpretation. CF and NC wrote the manuscript. All authors read and approved the final manuscript.

\section{Acknowledgments}

We are grateful to David Baillie, Fiona Brinkman, Carl Lowenberger, and Frederic Pio for valuable advice and discussions. We thank Duncan Napier, Martin Siegert, and Ata Roudgar for IT support and acknowledge Compute Canada for providing computational resources in form of the WestGrid grid computing facility. We further appreciate help from Spencer Myrtle for proofreading the manuscript. We also acknowledge the valuable input from all members of the Chen lab. The study was funded by NSERC of Canada (to NC) and the Simon Fraser University Community Trust Endowment Fund 
through the BCID Project. CF was supported by Weyerhaeuser and Simon Fraser University graduate scholarships.

Received: 15 January 2013 Accepted: 19 June 2013 Published: 27 June 2013

\section{References}

1. WHO: World Malaria Report 2010. Geneva, Switzerland: World Health Organization; 2010 [http://www.who.int/malaria/ world_malaria_report_2010/en/index.html]

2. Price RN, Tjitra E, Guerra CA, Yeung S, White NJ, Anstey NM: Vivax malaria: neglected and not benign. AmJTrop Med Hyg 2007, 77(6 Suppl):79-87.

3. Antinori S, Galimberti L, Milazzo L, Corbellino M: Plasmodium knowlesi: the emerging zoonotic malaria parasite. Acta Trop 2013, 125(2):191-201.

4. Olotu A, Fegan G, Wambua J, Nyangweso G, Awuondo KO, Leach A, Lievens M, Leboulleux D, Njuguna P, Peshu N, et al: Four-year efficacy of RTS, S/ AS01E and its interaction with malaria exposure. N Engl J Med 2013, 368(12):1111-1120.

5. The RTS SCTP: A Phase 3 Trial of RTS,S/AS01 Malaria Vaccine in African Infants. N Engl J Med 2012, 367:2284-2295. doi:10.1056/NEJMoa1208394. http://www.nejm.org/doi/full/10.1056/NEJMoa1208394.

6. Patarroyo MA, Calderon D, Moreno-Perez DA: Vaccines against Plasmodium vivax: a research challenge. Expert Rev Vaccines 2012, 11(10):1249-1260.

7. Carlton JM, Angiuoli SV, Suh BB, Kooij TW, Pertea M, Silva JC, Ermolaeva MD, Allen JE, Selengut JD, Koo HL, et al: Genome sequence and comparative analysis of the model rodent malaria parasite Plasmodium yoelii yoelii. Nature 2002, 419(6906):512-519.

8. Gardner MJ, Hall N, Fung E, White O, Berriman M, Hyman RW, Carlton JM, Pain A, Nelson KE, Bowman S, et al: Genome sequence of the human malaria parasite Plasmodium falciparum. Nature 2002, 419(6906):498-511.

9. Hall N, Karras M, Raine JD, Carlton JM, Kooij TW, Berriman M, Florens L, Janssen CS, Pain A, Christophides GK, et al: A comprehensive survey of the Plasmodium life cycle by genomic, transcriptomic, and proteomic analyses. Science 2005, 307(5706):82-86

10. Carlton JM, Adams JH, Silva JC, Bidwell SL, Lorenzi H, Caler E, Crabtree J, Angiuoli SV, Merino EF, Amedeo $P$, et al: Comparative genomics of the neglected human malaria parasite Plasmodium vivax. Nature 2008, 455(7214):757-763

11. Pain A, Bohme $U$, Berry AE, Mungall K, Finn RD, Jackson AP, Mourier T, Mistry J, Pasini EM, Aslett MA, et al: The genome of the simian and human malaria parasite Plasmodium knowlesi. Nature 2008, 455(7214):799-803.

12. Frech $\mathrm{C}$, Chen N: Genome comparison of human and non-human malaria parasites reveals species subset-specific genes potentially linked to human disease. PLoS Comput Biol 2011, 7(12):e1002320.

13. Recker M, Buckee CO, Serazin A, Kyes $S$, Pinches $R$, Christodoulou Z, springer AL, Gupta S, Newbold Cl: Antigenic variation in Plasmodium falciparum malaria involves a highly structured switching pattern. PLOS Pathog 2011, 7(3):e1001306.

14. Cunningham D, Lawton J, Jarra W, Preiser P, Langhorne J: The pir multigene family of Plasmodium: antigenic variation and beyond. Mol Biochem Parasitol 2010, 170(2):65-73.

15. Su XZ, Heatwole VM, Wertheimer SP, Guinet F, Herrfeldt JA, Peterson DS, Ravetch JA, Wellems TE: The large diverse gene family var encodes proteins involved in cytoadherence and antigenic variation of Plasmodium falciparum-infected erythrocytes. Cell 1995, 82(1):89-100.

16. Cheng Q, Cloonan N, Fischer K, Thompson J, Waine G, Lanzer M, Saul A: stevor and rif are Plasmodium falciparum multicopy gene families which potentially encode variant antigens. Mol Biochem Parasitol 1998 97(1-2):161-176

17. Winter G, Kawai S, Haeggstrom M, Kaneko O, Von Euler A, Kawazu S, Palm $D$, Fernandez V, Wahlgren M: SURFIN is a polymorphic antigen expressed on Plasmodium falciparum merozoites and infected erythrocytes. $J$ Exp Med 2005, 201(11):1853-1863.

18. Sam-Yellowe TY, Florens $L$, Johnson JR, Wang T, Drazba JA, Le Roch KG, Zhou Y, Batalov S, Carucci DJ, Winzeler EA, et al: A Plasmodium gene family encoding Maurer's cleft membrane proteins: structural properties and expression profiling. Genome Res 2004, 14(6):1052-1059.

19. Del Portillo HA, Fernandez-Becerra C, Bowman S, Oliver K, Preuss M, Sanchez CP, Schneider NK, Villalobos JM, Rajandream MA, Harris D, et al:
A superfamily of variant genes encoded in the subtelomeric region of Plasmodium vivax. Nature 2001, 410(6830):839-842.

20. Janssen CS, Phillips RS, Turner CM, Barrett MP: Plasmodium interspersed repeats: the major multigene superfamily of malaria parasites. Nucleic Acids Res 2004, 32(19):5712-5720.

21. Janssen CS, Barrett MP, Turner CM, Phillips RS: A large gene family for putative variant antigens shared by human and rodent malaria parasites. Proceedings 2002, 269(1489):431-436.

22. Al-Khedery B, Barnwell JW, Galinski MR: Antigenic variation in malaria: a 3' genomic alteration associated with the expression of a P. knowlesi variant antigen. Mol Cell 1999, 3(2):131-141.

23. Sargeant TJ, Marti M, Caler E, Carlton JM, Simpson K, Speed TP, Cowman AF: Lineage-specific expansion of proteins exported to erythrocytes in malaria parasites. Genome Biol 2006, 7(2):R12.

24. Hiller NL, Bhattacharjee S, Van Ooij C, Liolios K, Harrison T, Lopez-Estrano C, Haldar K: A host-targeting signal in virulence proteins reveals a secretome in malarial infection. Science 2004, 306(5703):1934-1937.

25. Marti M, Good RT, Rug M, Knuepfer E, Cowman AF: Targeting malaria virulence and remodeling proteins to the host erythrocyte. Science 2004, 306(5703):1930-1933.

26. Frech C, Chen N: Genome-wide comparative gene family classification. PLoS One 2010, 5(10):e13409.

27. Aurrecoechea C, Brestelli J, Brunk BP, Dommer J, Fischer S, Gajria B, Gao X, Gingle A, Grant G, Harb OS, et al: PlasmoDB: a functional genomic database for malaria parasites. Nucleic Acids Res 2009, 37:D539-D543. Database issue.

28. Loewenstein Y, Portugaly E, Fromer M, Linial M: Efficient algorithms for accurate hierarchical clustering of huge datasets: tackling the entire protein space. Bioinformatics 2008, 24(13):i41-i49.

29. Chen F, Mackey AJ, Stoeckert CJ Jr, Roos DS: OrthoMCL-DB: querying a comprehensive multi-species collection of ortholog groups. Nucleic Acids Res 2006, 34:D363-D368. Database issue.

30. Finn RD, Clements J, Eddy SR: HMMER web server: interactive sequence similarity searching. Nucleic Acids Res 2011, 39:W29-W37. Web Server issue.

31. Anantharaman $V$, lyer $L M$, Balaji $S$, Aravind $L$ : Adhesion molecules and other secreted host-interaction determinants in Apicomplexa: insights from comparative genomics. Int Rev Cytol 2007, 262:1-74.

32. Wasmuth J, Daub J, Peregrin-Alvarez JM, Finney CA, Parkinson J: The origins of apicomplexan sequence innovation. Genome Res 2009, 19(7):1202-1213.

33. Gough J, Karplus K, Hughey R, Chothia C: Assignment of homology to genome sequences using a library of hidden Markov models that represent all proteins of known structure. J Mol Biol 2001, 313(4):903-919.

34. Loewenstein $Y$, Linial $M$ : Connect the dots: exposing hidden protein family connections from the entire sequence tree. Bioinformatics 2008 24(16):i193-i199.

35. Lavazec C, Sanyal S, Templeton TJ: Expression switching in the stevor and Pfmc-2TM superfamilies in Plasmodium falciparum. Mol Microbiol 2007, 64(6):1621-1634

36. Tsarukyanova I, Drazba JA, Fujioka H, Yadav SP, Sam-Yellowe TY: Proteins of the Plasmodium falciparum two transmembrane Maurer's cleft protein family, PfMC-2TM, and the $130 \mathrm{kDa}$ Maurer's cleft protein define different domains of the infected erythrocyte intramembranous network. Parasitol Res 2009, 104(4):875-891.

37. Kall L, Krogh A, Sonnhammer EL: Advantages of combined transmembrane topology and signal peptide prediction--the Phobius web server. Nucleic Acids Res 2007, 35:W429-W432. Web Server issue.

38. Merino EF, Fernandez-Becerra C, Durham AM, Ferreira JE, Tumilasci VF, D'Arc-Neves J, Da Silva-Nunes M, Ferreira MU, Wickramarachchi T, UdagamaRandeniya $P$, et al: Multi-character population study of the vir subtelomeric multigene superfamily of Plasmodium vivax, a major human malaria parasite. Mol Biochem Parasitol 2006, 149(1):10-16.

39. Waller KL, Cooke BM, Nunomura W, Mohandas N, Coppel RL: Mapping the binding domains involved in the interaction between the Plasmodium falciparum knob-associated histidine-rich protein (KAHRP) and the cytoadherence ligand $\mathrm{P}$. falciparum erythrocyte membrane protein 1 (PfEMP1). J Biol Chem 1999, 274(34):23808-23813.

40. Mayer C, Slater L, Erat MC, Konrat R, Vakonakis I: Structural analysis of the Plasmodium falciparum erythrocyte membrane protein 1 (PfEMP1) intracellular domain reveals a conserved interaction epitope. J Biol Chem 2012, 287(10):7182-7189. 
41. Fernandez-Becerra C, Yamamoto MM, Vencio RZ, Lacerda M, Rosanas-Urgell A, Del Portillo HA: Plasmodium vivax and the importance of the subtelomeric multigene vir superfamily. Trends Parasitol 2009, 25(1):44-51.

42. Neafsey DE, Galinsky K, Jiang RH, Young L, Sykes SM, Saif S, Gujja S, Goldberg JM, Young S, Zeng Q, et al: The malaria parasite Plasmodium vivax exhibits greater genetic diversity than Plasmodium falciparum. Nat Genet 2012, 44(9):1046-1050.

43. Bozdech Z, Mok S, Hu G, Imwong M, Jaidee A, Russell B, Ginsburg H, Nosten F, Day NP, White NJ, et al: The transcriptome of Plasmodium vivax reveals divergence and diversity of transcriptional regulation in malaria parasites. Proc Natl Acad Sci USA 2008, 105(42):16290-16295.

44. Bustamante LY, Bartholdson SJ, Crosnier C, Campos MG, Wanaguru M, Nguon C, Kwiatkowski DP, Wright GJ, Rayner JC: A full-length recombinant Plasmodium falciparum PfRH5 protein induces inhibitory antibodies that are effective across common PfRH5 genetic variants. Vaccine 2012.

45. Crosnier C, Bustamante LY, Bartholdson SJ, Bei AK, Theron M, Uchikawa M, Mboup S, Ndir O, Kwiatkowski DP, Duraisingh MT, et al: Basigin is a receptor essential for erythrocyte invasion by Plasmodium falciparum. Nature 2011, 480(7378):534-537.

46. Kemp DJ, Coppel RL, Anders RF: Repetitive proteins and genes of malaria. Annu Rev Microbiol 1987, 41:181-208.

47. Scherf A, Lopez-Rubio JJ, Riviere L: Antigenic variation in Plasmodium falciparum. Annu Rev Microbiol 2008, 62:445-470.

48. Wiser MF, Giraldo LE, Schmitt-Wrede HP, Wunderlich F: Plasmodium chabaudi: immunogenicity of a highly antigenic glutamate-rich protein. Exp Parasitol 1997, 85(1):43-54.

49. Wunderlich F, Brenner $\mathrm{HH}$, Helwig M: Plasmodium chabaudi malaria: protective immunization with surface membranes of infected erythrocytes. Infect Immun 1988, 56(12):3326-3328.

50. Wunderlich F, Helwig M, Schillinger G, Speth V, Wiser MF: Expression of the parasite protein Pc90 in plasma membranes of erythrocytes infected with Plasmodium chabaudi. Eur J Cell Biol 1988, 47(2):157-164.

51. Fonager J, Pasini EM, Braks JA, Klop O, Ramesar J, Remarque EJ, Vroegrijk IO, Van Duinen SG, Thomas AW, Khan SM, et al: Reduced CD36-dependent tissue sequestration of Plasmodium-infected erythrocytes is detrimental to malaria parasite growth in vivo. J Exp Med 2012, 209(1):93-107.

52. Cernetich-Ott A, Daly TM, Vaidya AB, Bergman LW, Burns JM Jr: Remarkable stability in patterns of blood-stage gene expression during episodes of non-lethal Plasmodium yoelii malaria. Malar J 2012, 11(1):265.

53. Clark BJ, Wells J, King SR, Stocco DM: The purification, cloning, and expression of a novel luteinizing hormone-induced mitochondrial protein in MA-10 mouse Leydig tumor cells. Characterization of the steroidogenic acute regulatory protein (StAR). J Biol Chem 1994, 269(45):28314-28322.

54. Liu J, Rone MB, Papadopoulos V: Protein-protein interactions mediate mitochondrial cholesterol transport and steroid biosynthesis. J Biol Chem 2006, 281(50):38879-38893.

55. Sherman IW: Biochemistry of Plasmodium (malarial parasites). Microbiol Rev 1979, 43(4):453-495.

56. Maguire PA, Sherman IW: Phospholipid composition, cholesterol content and cholesterol exchange in Plasmodium falciparum-infected red cells. Mol Biochem Parasitol 1990, 38(1):105-112.

57. Sein KK, Aikawa M: The prime role of plasma membrane cholesterol in the pathogenesis of immune evasion and clinical manifestations of falciparum malaria. Medical hypotheses 1998, 51(2):105-110.

58. Deleersnijder W, Prasomsitti P, Tungpradubkul S, Hendrix D, HamersCasterman C, Hamers R: Structure of a Plasmodium chabaudi acidic phosphoprotein that is associated with the host erythrocyte membrane. Mol Biochem Parasitol 1992, 56(1):59-68

59. Templeton TJ: The varieties of gene amplification, diversification and hypervariability in the human malaria parasite, Plasmodium falciparum. Mol Biochem Parasitol 2009, 166(2):109-116.

60. Lavazec C, Sanyal S, Templeton TJ: Hypervariability within the Rifin, Stevor and Pfmc-2TM superfamilies in Plasmodium falciparum. Nucleic Acids Res 2006, 34(22):6696-6707.

61. Korir CC, Galinski MR: Proteomic studies of Plasmodium knowlesi SICA variant antigens demonstrate their relationship with P. falciparum EMP1. Infect Genet Evol 2006, 6(1):75-79.

62. Hora R, Bridges DJ, Craig A, Sharma A: Erythrocytic casein kinase II regulates cytoadherence of Plasmodium falciparum-infected red blood cells. J Biol Chem 2009, 284(10):6260-6269.
63. Oh SS, Voigt S, Fisher D, Yi SJ, LeRoy PJ, Derick LH, Liu S, Chishti AH: Plasmodium falciparum erythrocyte membrane protein 1 is anchored to the actin-spectrin junction and knob-associated histidine-rich protein in the erythrocyte skeleton. Mol Biochem Parasitol 2000, 108(2):237-247.

64. Voigt S, Hanspal M, LeRoy PJ, Zhao PS, Oh SS, Chishti AH, Liu SC: The cytoadherence ligand Plasmodium falciparum erythrocyte membrane protein 1 (PfEMP1) binds to the P. falciparum knob-associated histidinerich protein (KAHRP) by electrostatic interactions. Mol Biochem Parasitol 2000, 110(2):423-428.

65. Waller KL, Nunomura W, Cooke BM, Mohandas N, Coppel RL: Mapping the domains of the cytoadherence ligand Plasmodium falciparum erythrocyte membrane protein 1 (PfEMP1) that bind to the knobassociated histidine-rich protein (KAHRP). Mol Biochem Parasitol 2002, 119(1):125-129.

66. Glenister FK, Fernandez KM, Kats LM, Hanssen E, Mohandas N, Coppel RL, Cooke BM: Functional alteration of red blood cells by a megadalton protein of Plasmodium falciparum. Blood 2009, 113(4):919-928.

67. Hodder AN, Maier AG, Rug M, Brown M, Hommel M, Pantic I, Puig-de -Morales-Marinkovic M, Smith B, Triglia T, Beeson J, et al: Analysis of structure and function of the giant protein Pf332 in Plasmodium falciparum. Mol Microbiol 2009, 71(1):48-65.

68. Waller KL, Stubberfield LM, Dubljevic V, Buckingham DW, Mohandas N, Coppel RL, Cooke BM: Interaction of the exported malaria protein Pf332 with the red blood cell membrane skeleton. Biochim Biophys Acta 2010, 1798(5):861-871.

69. Benian GM, Kiff JE, Neckelmann N, Moerman DG, Waterston RH: Sequence of an unusually large protein implicated in regulation of myosin activity in C. elegans. Nature 1989, 342(6245):45-50.

70. She R, Chu JS, Uyar B, Wang J, Wang K, Chen N: genBlastG: using BLAST searches to build homologous gene models. Bioinformatics 2011, 27(15):2141-2143.

71. Uyar B, Chu JS, Vergara IA, Chua SY, Jones MR, Wong T, Baillie DL, Chen N: RNA-seq analysis of the C. briggsae transcriptome. Genome Res 2012, 22(8):1567-1580

72. Birney E, Clamp M, Durbin R: GeneWise and Genomewise. Genome Res 2004, 14(5):988-995.

73. Frith MC: A new repeat-masking method enables specific detection of homologous sequences. Nucleic Acids Res 2011, 39(4):e23.

74. Thompson JD, Gibson TJ, Higgins DG, et al: Multiple sequence alignment using ClustalW and ClustalX. In Current protocols in bioinformatics. Edited by Baxevanis AD. ; 2002. Chapter 2:Unit 23.

75. Di Tommaso P, Moretti S, Xenarios I, Orobitg M, Montanyola A, Chang JM, Taly JF, Notredame C: T-Coffee: a web server for the multiple sequence alignment of protein and RNA sequences using structural information and homology extension. Nucleic Acids Res 2011, 39:W13-W17. Web Server issue).

76. Nicholas KB, Nicholas HB Jr, Deerfield DW II: GeneDoc: Analysis and Visualization of Genetic Variation. EMBNEW.NEWS 1997, 4:14. http://www. nrbsc.org/gfx/genedoc/gdfeedb.htm.

77. Eddy SR: Accelerated Profile HMM Searches. PLoS Comput Biol 2011, 7(10):e1002195

78. Wilson D, Pethica R, Zhou Y, Talbot C, Vogel C, Madera M, Chothia C, Gough J: SUPERFAMILY-sophisticated comparative genomics, data mining, visualization and phylogeny. Nucleic Acids Res 2009, 37:D380-D386. Database issue.

79. Frech C, Choo C, Chen N: FeatureStack: Perl module for comparative visualization of gene features. Bioinformatics 2012 28(23):3137-3138

80. Roy A, Kucukural A, Zhang Y: I-TASSER: a unified platform for automated protein structure and function prediction. Nat Protoc 2010, 5(4):725-738.

81. Zhang Y: I-TASSER server for protein 3D structure prediction. BMC Bioinforma 2008, 9:40

82. Zhang Y, Skolnick J: TM-align: a protein structure alignment algorithm based on the TM-score. Nucleic Acids Res 2005, 33(7):2302-2309.

83. Krogh A, Larsson B, Von Heijne G, Sonnhammer EL: Predicting transmembrane protein topology with a hidden Markov model: application to complete genomes. J Mol Biol 2001, 305(3):567-580.

84. Cole C, Barber JD, Barton GJ: The Jpred 3 secondary structure prediction server. Nucleic Acids Res 2008, 36:W197-W201. Web Server issue.

85. Tamura K, Peterson D, Peterson N, Stecher G, Nei M, Kumar S: MEGA5: molecular evolutionary genetics analysis using maximum likelihood, 
evolutionary distance, and maximum parsimony methods. Mol Biol Evol 2011, 28(10):2731-2739.

86. Finn RD, Mistry J, Tate J, Coggill P, Heger A, Pollington JE, Gavin OL, Gunasekaran P, Ceric G, Forslund K, et al: The Pfam protein families database. Nucleic Acids Res 2010, 38:D211-D222. Database issue.

87. Carlton JM, Escalante AA, Neafsey D, Volkman SK: Comparative evolutionary genomics of human malaria parasites. Trends Parasitol 2008, 24(12):545-550.

doi:10.1186/1471-2164-14-427

Cite this article as: Frech and Chen: Variant surface antigens of malaria parasites: functional and evolutionary insights from comparative gene family classification and analysis. BMC Genomics 2013 14:427.

\section{Submit your next manuscript to BioMed Central and take full advantage of:}

- Convenient online submission

- Thorough peer review

- No space constraints or color figure charges

- Immediate publication on acceptance

- Inclusion in PubMed, CAS, Scopus and Google Scholar

- Research which is freely available for redistribution 